\title{
Bis-pyrimidine acetamides: design, synthesis and biological evaluation
}

\author{
Sanjiv Kumar², Siong Meng Lim, ${ }^{3,4}$, Kalavathy Ramasamy 3,4, Mani Vasudevan5, Syed Adnan Ali Shah 3,6 \\ and Balasubramanian Narasimhan ${ }^{1 *}$
}

\begin{abstract}
Background: In the past few years, increased resistance of microorganisms towards antimicrobial agents become a serious health problem, so there is a need for the discovery of new antimicrobial agents. On the other hand, bispyrimidines possess various types of biological activity. In view of this, in the present study we have designed and synthesized a new series of bis-pyrimidine acetamides by Claisen-Schmidt condensation and screened for its in vitro antimicrobial and anticancer activities.

Results: The synthesized bis-pyrimidine acetamide derivatives were confirmed by $\mathrm{IR},{ }^{1} \mathrm{H} /{ }^{13} \mathrm{C}-\mathrm{NMR}$, Mass spectral studies as well C, H, N analyses. The synthesized compounds were evaluated for their in vitro antimicrobial potential against Gram positive (Staphylococcus aureus and Bacillus subtilis); Gram negative (Escherichia coli, Pseudomonas aeruginosa and Salmonella enterica) bacterial and fungal (Candida albicans and Aspergillus niger) strains by tube dilution technique and the minimum inhibitory concentration (MIC) recorded in $\mu \mathrm{mol} / \mathrm{mL}$ was comparable to reference drugs, cefadroxil (antibacterial) and fluconazole (antifungal). The in vitro anticancer activity $\left({ } C_{50}\right.$ value) determined against human colorectal carcinoma (HCT116) cancer cell line by Sulforhodamine B (SRB) technique and 5-fluorouracil used as reference drug.

Conclusions: The biological study demonstrated that compounds $\mathbf{3}, \mathbf{1 3}, \mathbf{1 6}, \mathbf{1 7}$ and $\mathbf{1 8}$ were found to be most active antimicrobial agents with best MIC values than the cefadroxil (antibacterial) and fluconazole (antifungal) and compounds 12, 16 and $\mathbf{1 8}$ found to have better anticancer activity against human colorectal carcinoma (HCT116) cancer cell line with best $I C_{50}$ value than the 5 -fluorouracil (anticancer).
\end{abstract}

Keywords: Bis-pyrimidines, Claisen-Schmidt condensation, Antimicrobial, Anticancer, SAR

\section{Background}

The treatment of bacterial infections remains a challenging therapeutic problem because of emerging infectious diseases as well the increasing number of multidrug resistant microbial pathogens. Despite the many antimicrobial and chemotherapeutic agents are available in market, the emergence of old and new antibiotic resistant bacterial species in the last decade lead to a substantial need for the discovery of new classes of antimicrobial compounds [1]. Cancer is one of the most serious health problems all over the world and one of the leading causes

\footnotetext{
*Correspondence: naru2000us@yahoo.com

1 Faculty of Pharmaceutical Sciences, Maharshi Dayanand University,

Rohtak 124001, India

Full list of author information is available at the end of the article
}

of death. Thus, in the past for several decades, researchers have been struggling to find effective clinical approaches for the treatment of cancer and search for novel anticancer agents [2]. Among a wide variety of heterocyclic compounds that have been explored for developing medicinally important molecules, nitrogen containing heterocyclic pyrimidine derivatives occupies an important place in the field of medicinal chemistry [3]. The presence of a pyrimidine base in thymine, cytosine and uracil, which are the essential binding blocks of nucleic acids, DNA and RNA is one possible reason for their biological and therapeutical activities. Number of marketed drugs (Fig. 1) contains pyrimidine nucleus i.e. nilotinib (i) and capecitabine (ii) as anticancer; proquazone (iii) as anti-inflammatory; idoxuridine (iv) and trifluoridine (v) 
<smiles>Cc1ccc(C(=O)Nc2cc(-n3cncc3C)cc(C(F)(F)F)c2)cc1Nc1nccc(-c2ccncc2)n1</smiles><smiles>O=c1[nH]c(=O)n(C2COC(CO)C(O)C2)cc1C(F)(F)F</smiles>

(v)

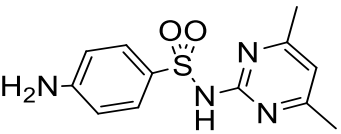

(ix)<smiles>CCCCOC(=O)Nc1nc(=O)n(C2OC(C)C(O)C2O)cc1F</smiles>

(ii)

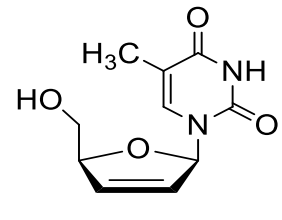

(vii)<smiles>Cc1ccc2c(-c3ccccc3)nc(=O)n(C(C)C)c2c1</smiles>

(iii)<smiles>O=c1[nH]c(=O)n(C2COCC(O)C2)cc1I</smiles>

(iv)<smiles></smiles>

(vi)<smiles>CCc1nc(N)nc(N)c1-c1ccc(Cl)cc1</smiles>

(x)



(xi)<smiles>COc1cc(Cc2cnc(N)nc2N)cc(OC)c1OC</smiles>

(viii)

Fig. 1 Some marketed drugs contains pyrimidine moiety

as antiviral; zidovudine (vi) and stavudine (vii) as antiHIV; trimethoprim (viii) and sulphamethiazine (ix) as antibacterial; pyrimethamine $(\mathrm{x})$ as antimalarial; minoxidil (xi) and ketanserin (xii) as antihypertensive [4]. The literature survey indicated that a wide range of pharmacological activities are exhibited by the compounds encompassing pyrimidine nucleus i.e. antibacterial [5], antifungal [6], antileishmanial [7], antimycobacterial [8], antimicrobial [9], anti-inflammatory [10], anticancer [11], antiviral [12], antitubercular [13], antimalarial [14], antioxidant activity [15], central nervous system (CNS) depressant and calcium channel blocker [8]. Based on the literature survey and biological profile of bis-pyrimidine acetamides summarized in Fig. 2 and in continuation of our efforts in searching novel antimicrobial and anticancer agents [16-18], in the present work, we hereby report the synthesis, antimicrobial and anticancer activities of a series of bis-pyrimidine acetamide derivatives.

\section{Results and discussion}

\section{Chemistry}

A general approach to synthesize the designed bispyrimidine acetamide derivatives based on ClaisenSchmidt condensation is outlined in Scheme 1. Initially, the bis-chalcone (I) was synthesized by the reaction of 1-(4-nitrophenyl)ethanone and terephthalaldehyde. The cyclization of a bis-chalcone into the corresponding bis-pyrimidine (II) was accomplished by the reaction of the bis-chalcone with guanidine nitrate. The reaction of bis-pyrimidine (II) with 2-chloroacetyl chloride resulted in the formation of III $\left\{N, N^{\prime}-\left(6,6^{\prime}-(1,4-\right.\right.$ phenylene $)$ bis(4-(4-nitrophenyl)pyrimidine-6,2-diyl))bis(2-chloroacetamide) $\}$ which on reaction with corresponding substituted aniline yielded the title compounds (1-20). The chemical structures of the synthesized compounds were established by the determination of their physicochemical and spectroscopic data (FT-IR, ${ }^{1} \mathrm{H}-\mathrm{NMR},{ }^{13} \mathrm{C}$ NMR, Mass) and elemental analyses. The structure of the bis-chalcone was confirmed by the corresponding IR (KBr pellets, $\mathrm{cm}^{-1}$ ). The IR spectrum of bis-chalcone (I) showed the characteristic band at $1690 \mathrm{~cm}^{-1}$ which indicated the presence of a $-\mathrm{C}=\mathrm{O}$ group and characteristic bands at 3106 and $1517 \mathrm{~cm}^{-1}$ indicated the presence of $\mathrm{C}-\mathrm{H}$ and $\mathrm{C}=\mathrm{C}$ group in aromatic ring respectively. The existence of $\mathrm{Ar}-\mathrm{NO}_{2}$ group in bis-chalcone (I) was displayed by the existence of symmetric $\mathrm{Ar}-\mathrm{NO}_{2}$ stretches in the scale of $1341 \mathrm{~cm}^{-1}$ and characteristic bands at 2906 and $1598 \mathrm{~cm}^{-1}$ indicated the presence of $\mathrm{C}-\mathrm{H}$ and $\mathrm{C}=\mathrm{C}$ group in alkyl chain respectively. Bis-pyrimidine (II) showed the characteristic bands at 3106 and $1518 \mathrm{~cm}^{-1}$ for the presence of $\mathrm{C}-\mathrm{H}$ and $\mathrm{C}=\mathrm{C}$ group in aromatic ring respectively and characteristic bands at 3370, 1600 and $1349 \mathrm{~cm}^{-1}$ for indicated the presence of $\mathrm{C}-\mathrm{NH}_{2}$; $\mathrm{N}=\mathrm{CH}$ str., of pyrimidine and $\mathrm{C}-\mathrm{N}$ sym stretches of $\mathrm{Ar}-\mathrm{NO}_{2}$ group. $N, N^{\prime}-\left(6,6^{\prime}-(1,4-P h e n y l e n e) b i s(4-(4-n i-\right.$ trophenyl)pyrimidine-6,2-diyl))bis(2-chloroacetamide) III showed appearance of IR stretching around 3105 and $1519 \mathrm{~cm}^{-1}$ in the spectral data for the presence of $\mathrm{C}-\mathrm{H}$ and $\mathrm{C}=\mathrm{C}$ group in aromatic ring respectively and characteristic bands at 1688, 1599, 2928, 761 and $1349 \mathrm{~cm}^{-1}$ 


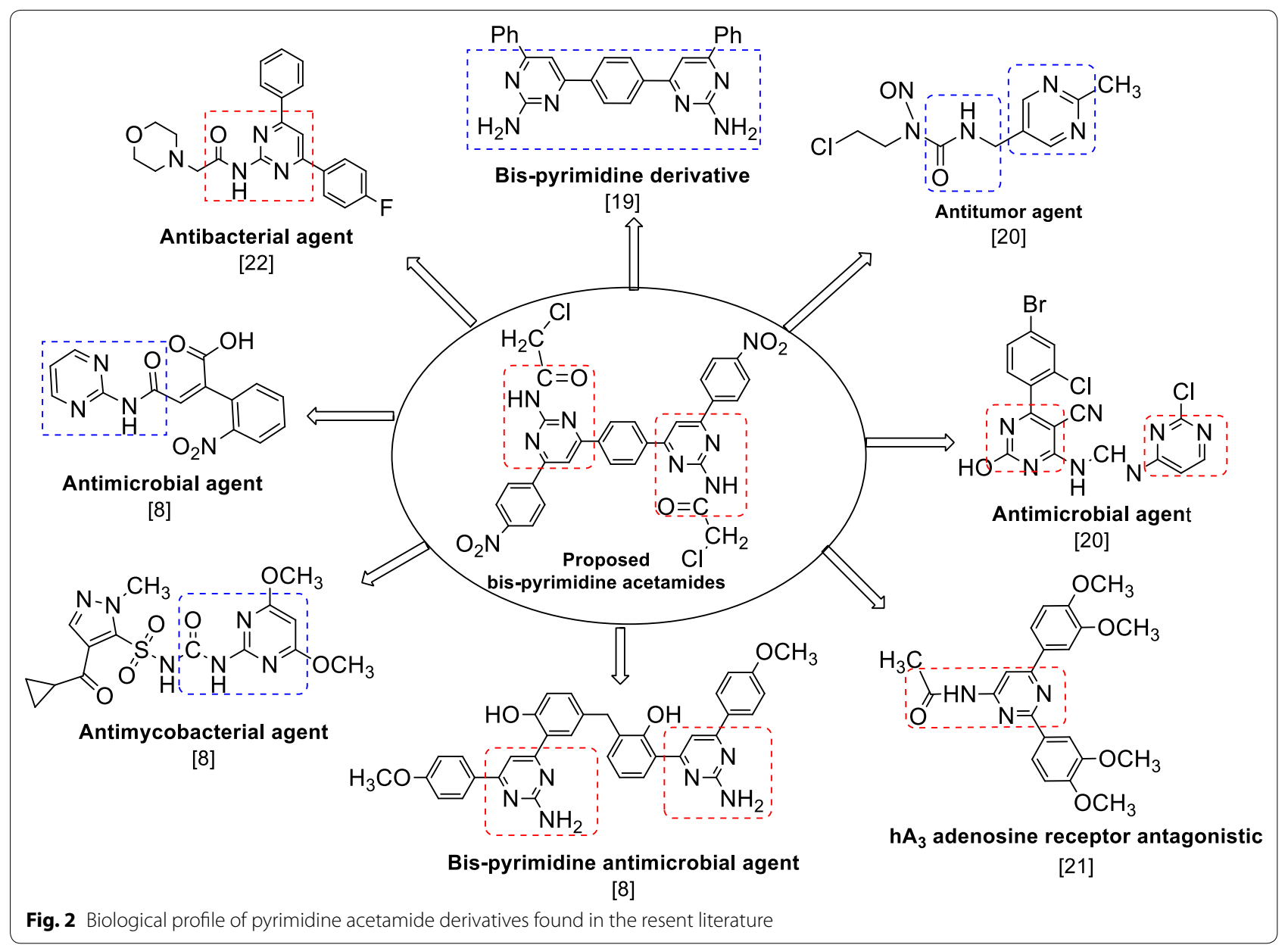

for the $\mathrm{NH}-\mathrm{C}=\mathrm{O} ; \mathrm{N}=\mathrm{CH}$ str., of pyrimidine; $\mathrm{C}-\mathrm{H}$ str., $\mathrm{CH}_{2} ; \mathrm{C}-\mathrm{Cl}$ str., ( $\mathrm{Ar}-\mathrm{Cl}$ ); $\mathrm{C}-\mathrm{N}$ sym str. of aromatic $\mathrm{NO}_{2}$ group respectively. The structure of the bis-chalcone and its cyclized products were further confirmed by the corresponding ${ }^{1} \mathrm{H}-\mathrm{NMR}$ spectra. The ${ }^{1} \mathrm{H}-\mathrm{NMR}$ spectrum of bis-chalcone I showed two doublets at $7.59 \mathrm{ppm}$ $(J=15.1 \mathrm{~Hz})$ and $8.06 \mathrm{ppm}(J=15.1 \mathrm{~Hz})$; indicating that the $\mathrm{CH}=\mathrm{CH}$ group in the enone linkage is in a transconformation. The ${ }^{1} \mathrm{H}-\mathrm{NMR}$ spectrum of intermediateII showed a multiplet signals between 7.43 and $8.40 \delta$, ppm confirming the cyclisation of the bis-chalcone to give bis-pyrimidine ring. The ${ }^{1} \mathrm{H}-\mathrm{NMR}$ spectrum of compound intermediate-II showed a sharp singlet at $6.99 \delta$, ppm due to the $\mathrm{NH}_{2}$ protons and it also showed a sharp singlet at $7.86 \delta$, ppm due to $\mathrm{HC}=\mathrm{C}$ group, which confirmed the cyclization of the bis-chalcone into a bispyrimidine ring. Intermediate (III) showed the multiplet signals between 7.54 and $8.93 \delta$, ppm in ${ }^{1} \mathrm{H}-\mathrm{NMR}$ spectra which is indicative of aromatic proton and also exhibited sharp singlet at 7.42, 8.10, $3.66 \delta$, ppm due to the presence of $\mathrm{HC}=\mathrm{C} ;-\mathrm{NH} ;-\mathrm{CH}_{2}$ respectively confirmed the conversion of the bis-pyrimidine into bis-pyrimidine acetamide. The appearance of IR stretching around 1575-1569, 3086-2851 and $1580-1600 \mathrm{~cm}^{-1}$ in the spectral data of synthesized derivatives (1-20) specified the existence of $\mathrm{C}=\mathrm{N}$ group of pyrimidine ring; $\mathrm{C}-\mathrm{H}$ and $\mathrm{C}=\mathrm{C}$ group respectively. The IR absorption band in the scale of $1106-1105 \mathrm{~cm}^{-1}$ corresponds to the $\mathrm{C}-\mathrm{F}$ stretching of aromatic-fluoro compounds (11 and 12) and $697-536 \mathrm{~cm}^{-1}$ corresponds to the $\mathrm{C}-\mathrm{Br}$ stretching of aromatic-bromo derivative (16). The existence of an arylalkyl ether category $\left(\mathrm{C}-\mathrm{O}-\mathrm{C}, \mathrm{Ar}-\mathrm{OCH}_{3}\right)$ in derivatives, 4, 19 and 20 are established by the existence of an IR symmetric stretches around $1107-1088 \mathrm{~cm}^{-1}$. Further, the existence of halogen group in compounds $(6,7,9$, 10, 13, 17 and 18) is indicated by the existence of Ar$\mathrm{Cl}$ stretching vibrations at $761-700 \mathrm{~cm}^{-1}$. The impression of IR stretching at $1692-1620 \mathrm{~cm}^{-1}$ in the spectra of specified the existence of $\mathrm{NH}-\mathrm{C}=\mathrm{O}$ group of synthesized derivatives. The multiplet signals between 6.74 and 9.08 $\delta$, ppm in ${ }^{1} \mathrm{H}$-NMR spectra is indicative of aromatic proton of synthesized derivatives (1-20). The compounds, 


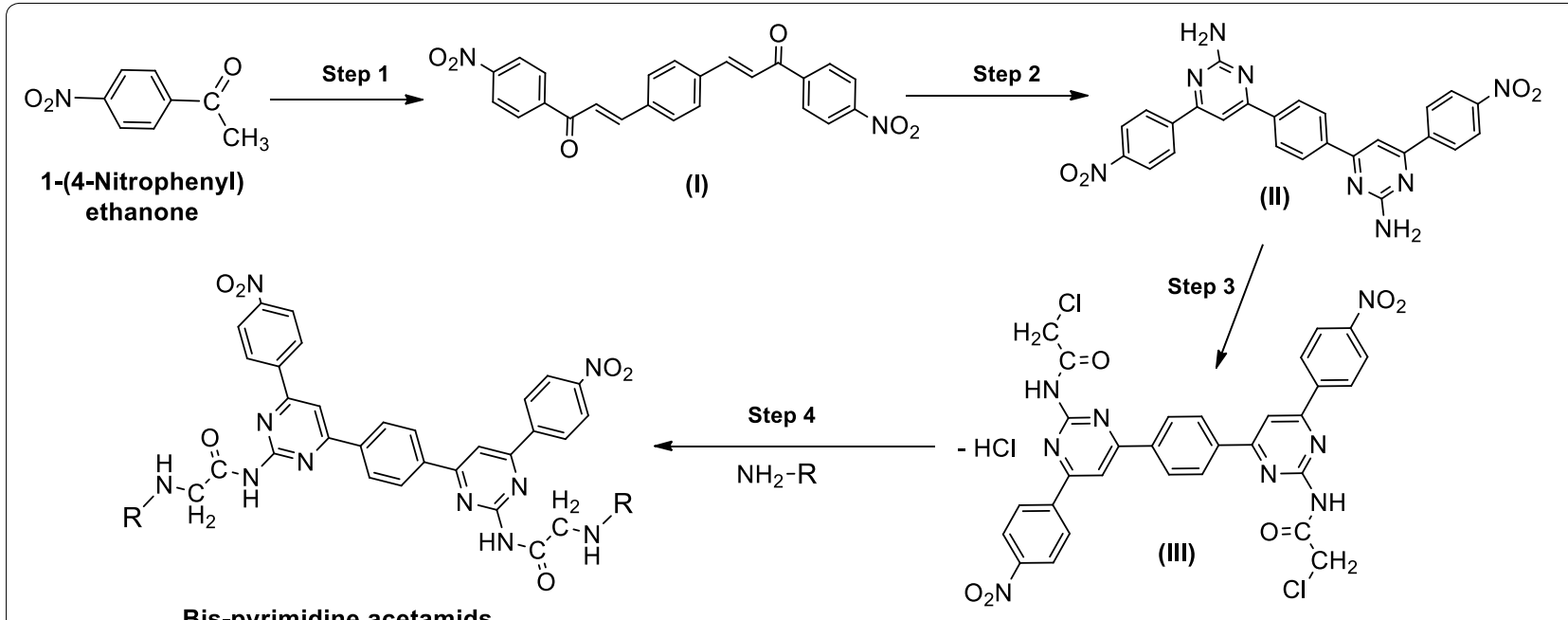

$(1-20)$

Step 1: Terephthalaldehyde, $\mathrm{NaOH}$, Methanol, Stirred 2-3 h, at room temp; Step 2: Guanidine nitrate, Methanol, $\mathrm{HCl}$, Reflux 5-6 h $\left(60^{\circ} \mathrm{C}\right)$ Step 3: Chloroacetyl chloride, Triethylamine, Methanol, Stirred at room temp./ Reflux 2-3 h $\left(30^{\circ} \mathrm{C}\right)$; Step 4: Substituted aniline, Methanol, Reflux $4-5 \mathrm{~h}\left(40^{\circ} \mathrm{C}\right)$

Comp.

Scheme 1 Synthesis of $N_{\text {, }} N^{\prime}$-(6,6'-(1,4-phenylene)bis(4-(4-nitrophenyl)pyrimidine-6,2-diyl)) bis(2-chloroacetamide) analogues

4, 19 and 20 showed singlet at $3.38-3.75 \delta$, ppm due to the presence of $\mathrm{OCH}_{3}$ of $\mathrm{Ar}-\mathrm{OCH}_{3}$ in their structure. All compounds showed singlet at 7.47-7.91 $\delta$, ppm due to the existence of $\mathrm{N}=\mathrm{CH}$ in pyrimidine ring. Synthesized compounds showed appearance of IR stretching around $1349 \mathrm{~cm}^{-1}$ which indicated the presence of $\mathrm{C}-\mathrm{N}$ sym str. of aromatic $\mathrm{NO}_{2}$ group. Compounds, 5, 14 and 15 showed singlet at $2.09-2.51 \delta$, ppm due to existence of $-\mathrm{CH}_{3}$ at ortho and para position. All compounds showed singlet at 3.34-3.38 $\delta, \mathrm{ppm}$ and $8.00-8.08 \delta$, ppm due to the existence of $-\mathrm{CH}_{2}-$ and $-\mathrm{NH}-$ groups respectively. The elemental analysis results of the synthesized bis-pyrimidine acetamide derivatives were found within $\pm 0.4 \%$ of the theoretical results. Finally, the ${ }^{13} \mathrm{C}$-NMR spectra of the bis-chalcone and the cyclized bis-pyrimidine were recorded in DMSO- $d_{6}$ and the spectral signals were in good agreement with the proposed molecular structure of the synthesized compounds. ${ }^{13} \mathrm{C}$-NMR 
spectra details of all compounds are given in the experimental part. Characteristic molecular ion peaks were observed in the mass spectra of the bis-chalcone and the cyclized bis-pyrimidine and final bis-pyrimidine acetamide derivatives.

\section{Antimicrobial activity}

The synthesized compounds were screened for their in vitro antimicrobial activity by tube dilution method. The investigation of antimicrobial screening revealed that some of the synthesized compounds showed moderate to good antimicrobial activity against Gram positive bacterial species: Staphylococcus aureus, Bacillus subtilis and Gram negative bacterial species: Escherichia coli, Pseudomonas aeruginosa, Salmonella enterica and fungal species: Aspergillus niger and Candida albicans. Particularly, compounds 3, 13, 16, 17 and 18 have shown more promising antimicrobial activity as compared to standard drugs cefadroxil (antibacterial) and fluconazole (antifungal). The remaining compounds displayed average to poor activity against all seven microbial species. In vitro antimicrobial activity results of synthesized compound are summarized in Table 1. Antibacterial screening results (Fig. 3) against Gram positive bacterial species demonstrated that compound $\mathbf{1 6}$ has showed good antibacterial activity against $S$. aureus and B. subtilis respectively. Compounds, 13 and $\mathbf{1 7}$ have sensible activity against $S$. aureus. The MIC values of $\mathbf{1 3}$ nearly close to MIC value of standard drugs cefadroxil while 16 and 17 have more active than standard drugs. Antibacterial screening results (Fig. 4) indicated that compound 3 possessed excellent activity against $S$. enterica. Compound 16 exhibited promising activity against $P$. aeruginosa and $E$. coli. Compound $\mathbf{1 7}$ was most active against $P$. aeruginosa. The MIC values of compounds $\mathbf{1 6}$ and $\mathbf{1 7}$ was more than the MIC values of standard drug cefadroxil Gram negative bacterial species. Antifungal screening results (Fig. 5) indicated that compound $\mathbf{3}$ and $\mathbf{1 8}$ were

Table 1 Antimicrobial and anticancer screening results of synthesized compounds

\begin{tabular}{|c|c|c|c|c|c|c|c|c|}
\hline \multirow[t]{4}{*}{ Compounds No. } & \multicolumn{7}{|c|}{ Minimum inhibitory concentration $(\mathrm{MIC}=\mu \mathrm{mol} / \mathrm{mL}$ ) } & \multirow{3}{*}{$\begin{array}{l}\mathrm{IC}_{50}(\mu \mathrm{mol} / \mathrm{mL}) \\
\text { Cancer cell line }\end{array}$} \\
\hline & \multicolumn{5}{|c|}{ Bacterial species } & \multirow{2}{*}{\multicolumn{2}{|c|}{ Fungal species }} & \\
\hline & \multicolumn{2}{|c|}{ Gram positive } & \multicolumn{3}{|l|}{ Gram negative } & & & \\
\hline & $\begin{array}{l}\text { S. aureus } \\
\text { MTCC-3160 }\end{array}$ & $\begin{array}{l}\text { B. subtilis } \\
\text { MTCC-441 }\end{array}$ & $\begin{array}{l}\text { P. aeruginosa } \\
\text { MTCC-3542 }\end{array}$ & $\begin{array}{l}\text { S. enterica } \\
\text { MTCC-1165 }\end{array}$ & $\begin{array}{l}\text { E. coli } \\
\text { MTCC-443 }\end{array}$ & $\begin{array}{l}\text { C. albicans } \\
\text { MTCC-227 }\end{array}$ & $\begin{array}{l}\text { A. niger } \\
\text { MTCC-281 }\end{array}$ & HCT-116 \\
\hline 1. & 0.72 & 0.36 & 1.45 & 0.72 & 1.45 & 1.45 & 1.45 & 3.86 \\
\hline 2. & 0.72 & 0.72 & 0.72 & 0.72 & 1.45 & 0.72 & 1.45 & 3.86 \\
\hline 3. & 1.31 & 0.33 & 0.66 & 0.66 & 1.31 & 0.66 & 1.31 & 3.50 \\
\hline 4. & 0.75 & 0.19 & 0.75 & 0.75 & 1.50 & 0.75 & 1.50 & 4.00 \\
\hline 5. & 0.78 & 0.78 & 0.78 & 1.56 & 1.56 & 0.78 & 1.56 & 4.16 \\
\hline 6. & 0.74 & 0.37 & 0.74 & 1.48 & 1.48 & 1.48 & 1.48 & 3.96 \\
\hline 7. & 0.67 & 0.67 & 1.34 & 0.67 & 1.34 & 1.34 & 1.34 & 3.58 \\
\hline 8. & 0.72 & 1.45 & 1.45 & 0.72 & 1.45 & 0.72 & 1.45 & 3.86 \\
\hline 9. & 0.74 & 0.74 & 0.74 & 1.48 & 1.48 & 0.74 & 1.48 & 1.98 \\
\hline 10. & 0.74 & 0.19 & 0.74 & 1.48 & 1.48 & 0.74 & 1.48 & 3.96 \\
\hline 11. & 0.39 & 0.39 & 0.77 & 0.77 & 0.77 & 0.77 & 1.55 & 1.52 \\
\hline 12. & 0.39 & 0.39 & 0.77 & 0.77 & 1.55 & 0.77 & 1.55 & 0.74 \\
\hline 13. & 0.17 & 0.34 & 0.67 & 0.67 & 1.34 & 0.67 & 1.34 & 3.58 \\
\hline 14. & 1.51 & 0.75 & 0.38 & 1.51 & 1.51 & 0.75 & 1.51 & 4.02 \\
\hline 15. & 0.38 & 0.38 & 0.75 & 0.75 & 0.75 & 0.75 & 1.51 & 2.17 \\
\hline 16. & 0.17 & 0.17 & 0.34 & 0.67 & 0.67 & 0.67 & 1.34 & 0.98 \\
\hline 17. & 0.17 & 0.34 & 0.34 & 0.69 & 1.37 & 0.69 & 1.37 & 1.22 \\
\hline 18. & 0.34 & 0.69 & 0.69 & 0.69 & 1.37 & 0.34 & 1.37 & 0.73 \\
\hline 19. & 0.75 & 0.38 & 0.75 & 0.75 & 1.50 & 0.75 & 1.50 & 4.00 \\
\hline 20. & 0.19 & 0.38 & 0.75 & 0.75 & 1.50 & 0.75 & 1.50 & 2.16 \\
\hline DMSO & 0.00 & 0.00 & 0.00 & 0.00 & 0.00 & 0.00 & 0.00 & - \\
\hline Cefadroxil & 1.72 & 0.86 & 1.72 & 1.72 & 1.72 & - & - & - \\
\hline Fluconazole & - & - & - & - & - & 1.02 & 2.04 & - \\
\hline 5-Fluorouracil & - & - & - & - & - & - & - & 7.67 \\
\hline
\end{tabular}



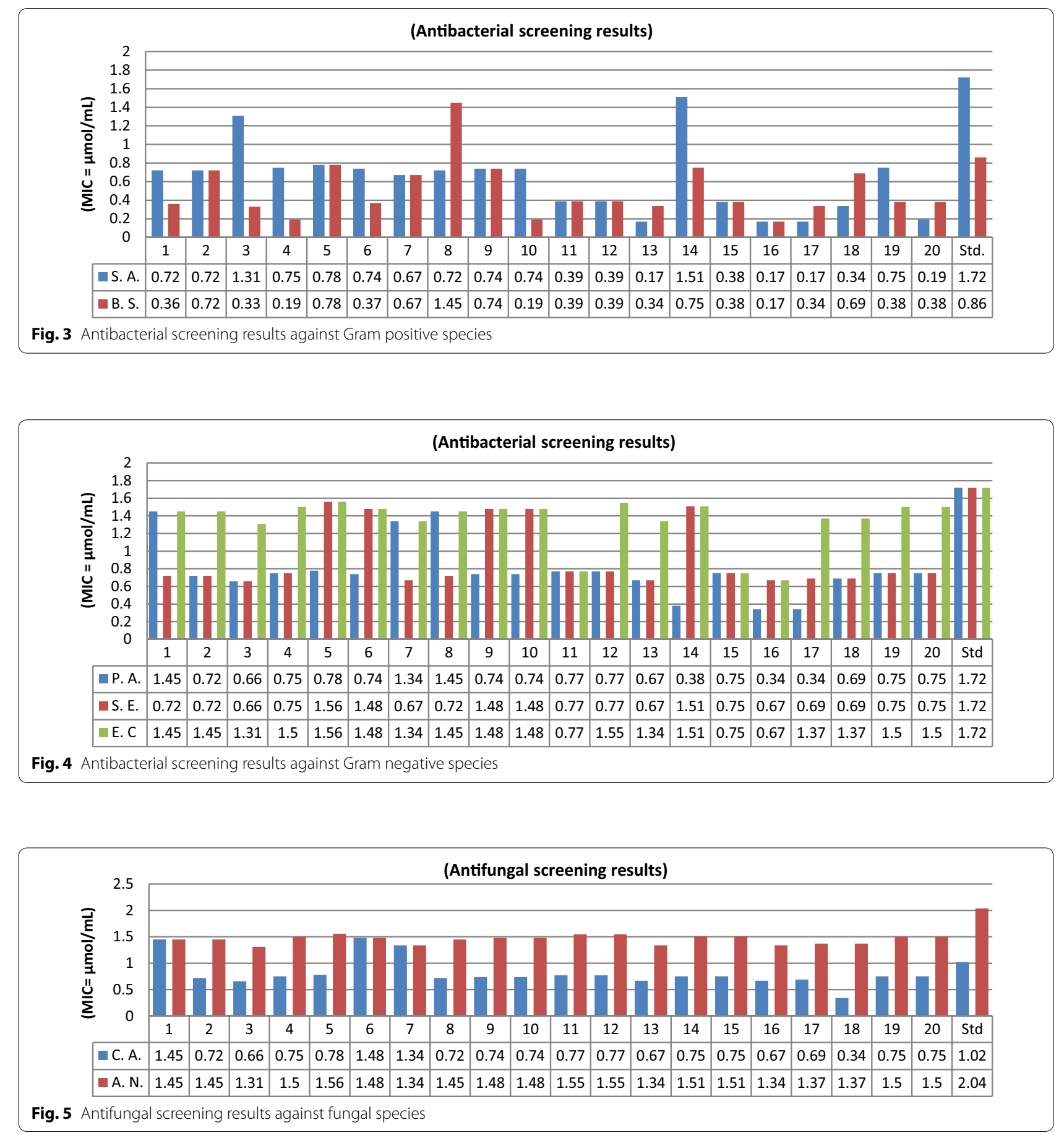

found to be most effective ones against $A$. niger and $C$. albicans respectively. The rest of the compounds of the series exhibited average to poor antifungal activity. The antimicrobial activity results of synthesized bis-pyrimidine acetamide derivatives indicated that the synthesized compounds are more active than the standard drugs and may be taken as a lead compound to discover novel antimicrobial agents.

\section{Anticancer activity}

The anticancer screening $\left(\mathrm{IC}_{50}=\mu \mathrm{mol} / \mathrm{mL}\right)$ of the synthesized bis-pyrimidine acetamide derivatives was 
determined against human colorectal carcinoma [HCT116 (ATCC CCL-247)] cancer cell line by Sulforhodamine B (SRB) technique [28] using 5-fluorouracil as reference drug and the results are presented in Table 1. Anticancer screening results (Fig. 6) revealed that in general bis-pyrimidine acetamides exhibited good anticancer potential against human colorectal cancer cell line, especially, Compounds, 12, 16 and 18 displayed more anticancer activity than the reference drug 5 -fluorouracil $\left(\mathrm{IC}_{50}=7.67 \mu \mathrm{mol} / \mathrm{mL}\right)$ with $\mathrm{IC}_{50}$ values of $0.74,0.98$ and $0.73 \mu \mathrm{mol} / \mathrm{mL}$ respectively.

The structure-activity relationship of the synthesized bis-pyrimidine acetamides indicated that the compounds bearing electron withdrawing group at different position of phenyl group plays an important role in enhancing the antimicrobial and anticancer potentials (Fig. 7).

\section{Experimental part}

Preparatory materials for the research work were obtained from commercial sources and were used without further purification. All reactions were monitored by thin-layer chromatography on $0.25 \mathrm{~mm}$ silica gel (Merck) plates, using benzene as mobile phase and spots were observed by exposure to iodine vapours or visualized with UV light. Melting points of synthesized compounds was determined in open capillary tube. An infrared spectrum was recorded (KBr-pellets) in Bruker 12060280, Software: OPUS 7.2.139.1294 spectrometer. ${ }^{1} \mathrm{H}-\mathrm{NMR}$ and ${ }^{13} \mathrm{C}$-NMR were recorded at 600 and $150 \mathrm{MHz}$, respectively on Bruker Avance III 600 NMR spectrometer by appropriate deuterated solvents. The results are conveyed in parts per million $(\delta, \mathrm{ppm})$ downfield from tetramethyl silane (internal standard). ${ }^{1} \mathrm{H}-\mathrm{NMR}$ spectral details of the synthesized derivatives are represented with multiplicity like singlet $(\mathrm{s})$; doublet $(\mathrm{d})$; triplet $(\mathrm{t})$; multiplet $(\mathrm{m})$ and the number hydrogen ion. Elemental analysis of the new synthesized compounds was obtained by Perkin-Elmer $2400 \mathrm{C}, \mathrm{H}$ and $\mathrm{N}$ analyzer. All the compounds gave $\mathrm{C}, \mathrm{H}$ and $\mathrm{N}$ analysis within $\pm 0.4 \%$ of the theoretical results. Mass spectra were taken on Waters Micromass Q-ToF Micro instrument. The synthesized

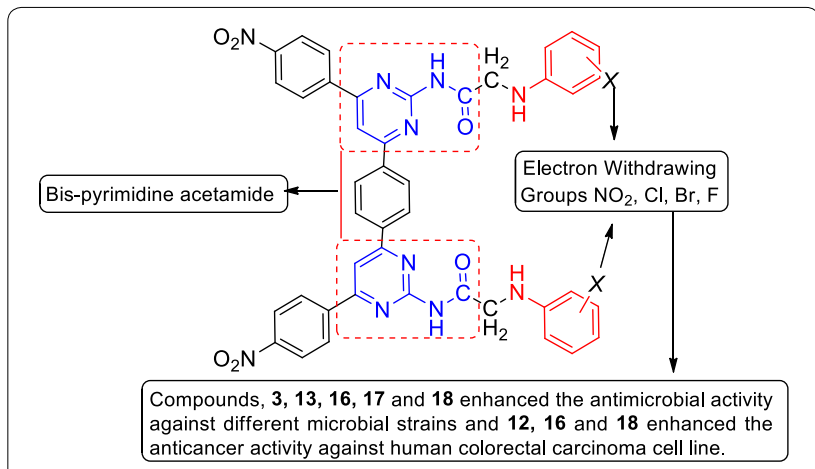

Fig. 7 Structure activity relationship of bis-pyrimidine acetamides

compounds were characterized by the determination of their physicochemical and spectral characteristics.

\section{General procedure of the synthesized bis-pyrimidine acetamide derivatives (1-20)}

Step i: Synthesis of 3,3'-(1,4-phenylene)bis(1-(4-nitrophenyl)prop-2-en-1-one) (I) A mixture of 1-(4-nitrophenyl) ethanone $(0.02 \mathrm{~mol})$ and terephthalaldehyde $(0.01 \mathrm{~mol})$ in methanol $(5-10 \mathrm{~mL})$ was stirred with drop wise addition of $40 \%$ sodium hydroxide solution $(10 \mathrm{~mL})$ at room temperature till a dark yellow mass was obtained ( $2-3 \mathrm{~h}$ ). Then reaction mixture was allowed to stand overnight at room temperature and then was poured into icecold water and acidified with hydrochloric acid and the precipitated 3,3'-(1,4-phenylene)bis(1-(4-nitrophenyl) prop-2-en-1-one) was filtered, dried and recrystallized from methanol [23, 24].

Step ii: Synthesis of 6,6'-(1,4-phenylene)bis(4-(4-nitrophenyl)pyrimidin-2-amine) (II) The solution of 3,3'-(1,4-phenylene)bis(1-(4-nitrophenyl)prop-2-en-1one) $(0.01 \mathrm{~mol})$ (synthesized in previous step-i) in methanol $(80 \mathrm{~mL})$ was added with $0.01 \mathrm{~mol}$ of potassium hydroxide and $40 \mathrm{~mL}$ of $0.50 \mathrm{M}$ solution of guanidine nitrate and refluxed for 5-6 h. The reaction mixture was then cooled and acidified with few drops of hydrochloric acid ( $20 \mathrm{~mL}$ of $0.5 \mathrm{M}$ solution) and the resultant precipi-

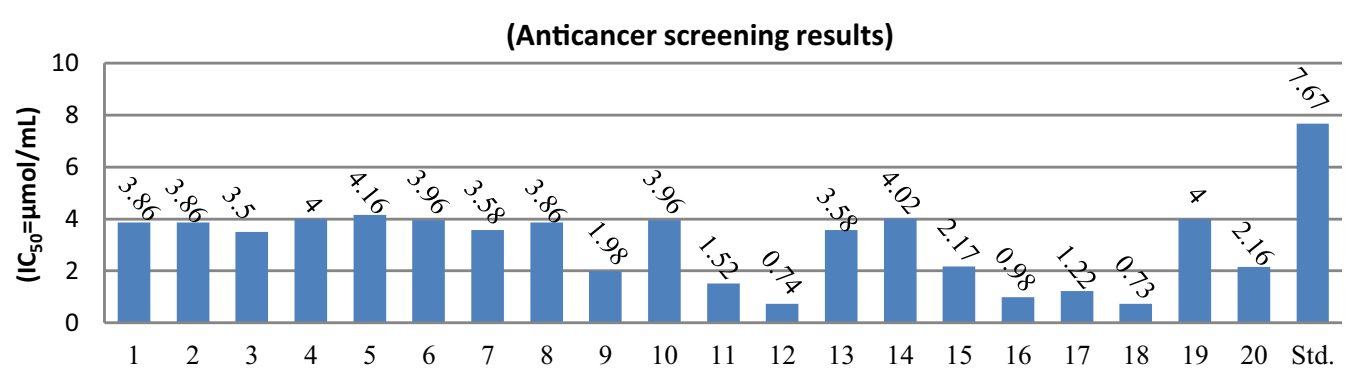

Fig. 6 Anticancer screening results of the synthesized compounds 
tate, 6,6'-(1,4-phenylene)bis(4-(4-nitrophenyl) pyrimidin2 -amine) was separated dried and recrystallized from methanol [23, 24].

Step iii: Synthesis of $N, N^{\prime}-\left(6,6^{\prime}-(1,4-p h e n y l e n e) b i s(4-(4-n i-\right.$ trophenyl)pyrimidine-6,2-diyl))bis (2-chloroacetamide) (III) In ethanol (30 mL), chloroacetyl chloride (0.02 mol) and 2-3 drops of triethylamine were added and the mixture was stirred in water bath for $10 \mathrm{~min}$ after that the solution of 6,6'-(1,4-phenylene)bis(4-(4-nitrophenyl) pyrimidin-2-amine) (synthesized in previous step-ii) $(0.01 \mathrm{~mol})$ in ethanol $(80 \mathrm{~mL})$ was added drop wise and refluxed for $2-3 \mathrm{~h}$. The reaction mixture was then cooled and poured into icecold water and resultant precipitate was filtered and washed with water, dried and recrystallized from alcohol [25].

Step iv: Synthesis of final (1-20) bis-pyrimidine acetamide derivatives The reaction mixture of $N, N^{\prime}-\left(6,6^{\prime}-(1,4-\right.$ phenylene)bis(4-(4-nitrophenyl)pyrimidine-6,2-diyl)) bis(2-chloroacetamide) ( $0.01 \mathrm{~mol})$ and substituted aniline $(0.02 \mathrm{~mol})$ in ethanol was refluxed for $4-5 \mathrm{~h}$. The reaction progress was monitored by thin layer chromatography. After completion of reaction, the reaction mixture was poured into icecold water and the precipitated title compound was filtered, dried and recrystallized from methanol.

\section{Spectral data interpretation of the synthesized compounds (Fig. 8)}

3,3'-(1,4-Phenylene)bis(1-(4-nitrophenyl)prop-2-en-1-one) (I) IR ( $\mathrm{KBr}$ pellets, $\left.\mathrm{cm}^{-1}\right)$ : $3106(\mathrm{C}-\mathrm{H}$ str., phenyl nucleus), 1517 ( $\mathrm{C}=\mathrm{C}$ str., phenyl nucleus), 1690 ( $\mathrm{C}=\mathrm{O}$ str.), 1598 ( $\mathrm{C}=\mathrm{C}$ str., alkyl chain), 2906 ( $\mathrm{C}-\mathrm{H}$ str., alkyl chain), 1341 (C-N sym str., Ar- $\left.\mathrm{NO}_{2}\right) ;{ }^{1} \mathrm{H}-\mathrm{NMR}\left(\delta\right.$, DMSO- $\left.d_{6}\right)$ : 6.94-9.08 $\left\{\mathrm{m}, 12 \mathrm{H}, \mathrm{Ar}=\mathrm{H}-2, \mathrm{H}-3, \mathrm{H}-5, \mathrm{H}-6,\left(\mathrm{H}-2^{\prime \prime}, \mathrm{H}-3^{\prime \prime}\right.\right.$, $\left.\left.\mathrm{H}-5^{\prime \prime}, \mathrm{H}-6^{\prime \prime}\right) \times 2\right\}, 7.59\left\{\mathrm{~d}, 4 \mathrm{H},(\mathrm{CH})_{2}, J=15.1 \mathrm{~Hz}\right), 8.06$ $\left(\mathrm{d}, 4 \mathrm{H},(\mathrm{CH})_{2}, J=15.1 \mathrm{~Hz}\right.$ of $\left.\mathrm{CH}_{2}=\mathrm{CH}_{2}\right\} ;{ }^{13} \mathrm{C}-\mathrm{NMR}(\delta$, DMSO- $\left.d_{6}\right)$ : 129.8 (C-2, C-3, C-5, C-6), 133.4 (C-1, C-4); $148.0\left(\mathrm{C}-1^{\prime}\right), 121.3\left(\mathrm{C}-2^{\prime}\right), 164.7(\mathrm{C}=\mathrm{O}) ; 145.1\left(\mathrm{C}-1^{\prime \prime}\right)$, 130.0 (C-2", C-6"), 124.9 (C-3", C-5"), 162.5 (C-4"); Elem. Anal. Calcd. $\mathrm{C}_{24} \mathrm{H}_{16} \mathrm{~N}_{2} \mathrm{O}_{6}$ : C, 67.29; H, 3.76; N, 6.54; Found: C, 67.26; H, 3.75; N, 6.50; MS ES+(ToF): $m / z 429$ $\left[\mathrm{M}^{+}+1\right]$.

6, 6'-(1,4-Phenylene)bis(4-(4-nitrophenyl)pyrimidin-2-amine) (II) IR ( $\mathrm{KBr}$ pellets, $\left.\mathrm{cm}^{-1}\right): 3107(\mathrm{C}-\mathrm{H}$ str., phenyl nucleus), 1518 ( $\mathrm{C}=\mathrm{C}$ str., phenyl nucleus), $3370\left(\mathrm{C}-\mathrm{NH}_{2}\right.$ str.), $1600(\mathrm{~N}=\mathrm{CH}$ str., of pyrimidine), $1349\left(\mathrm{C}-\mathrm{N}\right.$ sym str., $\left.\mathrm{Ar}-\mathrm{NO}_{2}\right) ;{ }^{1} \mathrm{H}-\mathrm{NMR}\left(\delta\right.$, DMSO- $\left.d_{6}\right)$ : 7.43-8.40 $\left\{\mathrm{m}, 12 \mathrm{H}, \mathrm{Ar}=\mathrm{H}-2, \mathrm{H}-3, \mathrm{H}-5, \mathrm{H}-6,\left(\mathrm{H}-2^{\prime \prime}, \mathrm{H}-3^{\prime \prime}\right.\right.$, $\left.\left.\mathrm{H}-5^{\prime \prime}, \mathrm{H}-6^{\prime \prime}\right) \times 2\right\}, 7.86\left(\mathrm{~s}, 2 \mathrm{H},(\mathrm{CH})_{2}\right.$ of pyrimidine $), 6.97$ $\left(\mathrm{s}, 2 \mathrm{H},(\mathrm{NH})_{2}\right) ;{ }^{13} \mathrm{C}-\mathrm{NMR}\left(\delta\right.$, DMSO- $\left.d_{6}\right): 128.5(\mathrm{C}-2, \mathrm{C}-3$,

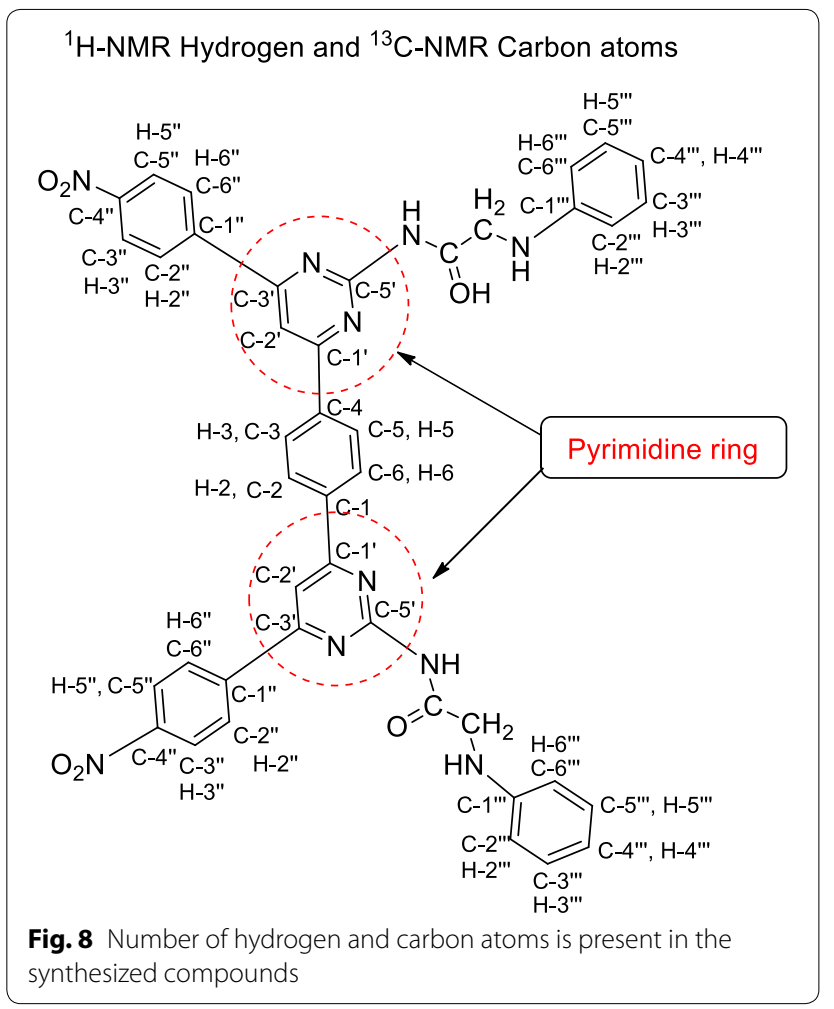

C-5, C-6), 134.6 (C-1, C-4); 157.8 (C-1', C-3', C-5'), 102 $\left(\mathrm{C}-2^{\prime}\right), 149.7\left(\mathrm{C}-5^{\prime}\right)$ pyrimidine; $141.5\left(\mathrm{C}-1^{\prime \prime}\right), 126.4\left(\mathrm{C}-2^{\prime \prime}\right.$, C-6"), 124.4 (C-3", C-5"), 148.3 (C-4"); Elem. Anal. Calcd. $\mathrm{C}_{26} \mathrm{H}_{18} \mathrm{~N}_{8} \mathrm{O}_{4}$ : C, 61.66; H, 3.58; N, 22.12; Found: C, 61.64; $\mathrm{H}, 3.51 ; \mathrm{N}, 22.15 ; \mathrm{MS}$ ES + (ToF): $m / z 507\left[\mathrm{M}^{+}+1\right]$.

$N, N^{\prime}-\left(6,6^{\prime}-(1,4-P h e n y l e n e) b i s(4-(4-n i t r o p h e n y l) p y r i m i-\right.$ dine-6,2-diyl))bis(2-chloroacetamide) (III) IR (KBr pellets, $\mathrm{cm}^{-1}$ ): 3105 (C-H str., phenyl nucleus), 1519 ( $\mathrm{C}=\mathrm{C}$ str., phenyl nucleus), 1688 ( $\mathrm{NH}-\mathrm{C}=\mathrm{O}$ str.), $1599(\mathrm{~N}=\mathrm{CH}$ str., of pyrimidine), $2928\left(\mathrm{C}-\mathrm{H}\right.$ str., $\left.\mathrm{CH}_{2}\right), 761(\mathrm{C}-\mathrm{Cl}$ str., Ar-Cl), 1349 (C-N sym. str., Ar- $\left.\mathrm{NO}_{2}\right) ;{ }^{1} \mathrm{H}-\mathrm{NMR}$ $\left(\delta, \mathrm{DMSO}^{-} d_{6}\right): 7.54-8.93\{\mathrm{~m}, 12 \mathrm{H}, \mathrm{Ar}=\mathrm{H}-2, \mathrm{H}-3, \mathrm{H}-5$, $\left.\mathrm{H}-6,\left(\mathrm{H}-2^{\prime \prime}, \mathrm{H}-3^{\prime \prime}, \mathrm{H}-5^{\prime \prime}, \mathrm{H}-6^{\prime \prime}\right) \times 2\right\}, 7.42\left(\mathrm{~s}, 2 \mathrm{H},(\mathrm{CH})_{2}\right.$ of pyrimidine), $8.10\left(\mathrm{~s}, 2 \mathrm{H},(\mathrm{NH})_{2}\right), 3.66\left(\mathrm{~s}, 4 \mathrm{H},\left(\mathrm{CH}_{2}\right)_{2}\right)$; ${ }^{13} \mathrm{C}-\mathrm{NMR}\left(\delta, \mathrm{DMSO}-d_{6}\right): 128.5(\mathrm{C}-2, \mathrm{C}-3, \mathrm{C}-5, \mathrm{C}-6), 136.6$ (C-1, C-4); 162.0 (C-1', C-3'), 108.0 (C-2'), 149.7 (C-5') pyrimidine; $141.0\left(\mathrm{C}-1^{\prime \prime}\right), 126.6\left(\mathrm{C}-2^{\prime \prime}, \mathrm{C}-6^{\prime \prime}\right), 124.5\left(\mathrm{C}-3^{\prime \prime}\right.$, C-5"), $149.8\left(\mathrm{C}-4^{\prime \prime}\right), 188.0(\mathrm{C}=\mathrm{O}), 44.3\left(\mathrm{CH}_{2}\right)$; Elem. Anal. Calcd. $\mathrm{C}_{30} \mathrm{H}_{20} \mathrm{Cl}_{2} \mathrm{~N}_{8} \mathrm{O}_{6}$ : C, 54.64; H, 3.06; N, 16.99; Found: C, 54.60; H, 3.00; N, 16.92; MS ES + (ToF): $m / z 660\left[\mathrm{M}^{+}\right.$ $+1]$.

$N, N^{\prime}-\left(6,6^{\prime}-(1,4-P h e n y l e n e) b i s(4-(4-n i t r o p h e n y l) p y r i m i-\right.$ dine-6,2-diyl))bis(2-((3-nitrophenyl) amino)acetamide) (1) Brown crystals; Yield: $70.12 \%$; M.p.: $252-254{ }^{\circ} \mathrm{C}$; R $f$ value: 90.12; IR ( $\left.\mathrm{KBr}, \mathrm{cm}^{-1}\right)$ : 3085 (C-H str., phenyl nucleus), 
1528 ( $\mathrm{C}=\mathrm{C}$ str., phenyl nucleus), 1679 (NH-C=O str.), $1572\left(\mathrm{~N}=\mathrm{CH}\right.$ str., of pyrimidine), $2863\left(\mathrm{C}-\mathrm{H}\right.$ str., $\left.\mathrm{CH}_{2}\right)$, 1217 (C-NH-str.), 1347 (C-N sym. str., Ar- $\left.\mathrm{NO}_{2}\right) ;{ }^{1} \mathrm{H}-$ NMR $\left(\delta\right.$, DMSO- $\left.d_{6}\right): 7.63-8.25\{\mathrm{~m}, 20 \mathrm{H}, \mathrm{Ar}=\mathrm{H}-2, \mathrm{H}-3$, $\mathrm{H}-5, \mathrm{H}-6,\left(\mathrm{H}-2^{\prime \prime}, \mathrm{H}-3^{\prime \prime}, \mathrm{H}-5^{\prime \prime}, \mathrm{H}-6^{\prime \prime}\right) \times 2,\left(\mathrm{H}-2^{\prime \prime \prime}, \mathrm{H}-4^{\prime \prime \prime}, \mathrm{H}-5^{\prime \prime \prime}\right.$, $\left.\left.\mathrm{H}-6^{\prime \prime \prime}\right) \times 2\right\}, 7.63\left(\mathrm{~s}, 2 \mathrm{H},(\mathrm{CH})_{2}\right.$ of pyrimidine), $8.08(\mathrm{~s}, 2 \mathrm{H}$, $\left.(\mathrm{NH})_{2}\right), 4.0\left(\mathrm{~s}, 2 \mathrm{H},(\mathrm{NH})_{2}\right), 3.40\left(\mathrm{~s}, 4 \mathrm{H},\left(\mathrm{CH}_{2}\right)_{2}\right) ;{ }^{13} \mathrm{C}-\mathrm{NMR}$ $\left(\delta\right.$, DMSO- $\left.d_{6}\right): 128.3,128.4(\mathrm{C}-2, \mathrm{C}-3, \mathrm{C}-5, \mathrm{C}-6), 136.5$ (C-1, C-4); 165.0 (C-1', C-3'), 109 (C-2'), 149.7 (C-5') pyrimidine; $141.0\left(\mathrm{C}-1^{\prime \prime}\right), 126.5\left(\mathrm{C}-2^{\prime \prime}, \mathrm{C}-6^{\prime \prime}\right), 124.5\left(\mathrm{C}-3^{\prime \prime}\right.$, C-5"), $149.6\left(\mathrm{C}-4^{\prime \prime}\right) ; 149.5\left(\mathrm{C}-1^{\prime \prime \prime}, \mathrm{C}-3^{\prime \prime \prime}\right), 106.9\left(\mathrm{C}-2^{\prime \prime \prime}\right)$, 129.9 (C-5"'), $122.1\left(\mathrm{C}-6^{\prime \prime \prime}\right) ; 170.2(\mathrm{C}=\mathrm{O}), 48.0\left(\mathrm{CH}_{2}\right)$; Elem. Anal. Calcd. $\mathrm{C}_{42} \mathrm{H}_{30} \mathrm{~N}_{12} \mathrm{O}_{10}$ : C, 58.47; H, 3.50; N, 19.48; Found: C, 58.42; H, 3.49; N, 19.46; MS ES + (ToF): $m / z 864\left[\mathrm{M}^{+}+1\right]$.

$N, N^{\prime}-\left(6,6{ }^{\prime}-(1,4-P h e n y l e n e) b i s(4-(4-n i t r o p h e n y l) p y r i m i-\right.$ dine-6,2-diyl))bis(2-((4-nitrophenyl) amino)acetamide)

(2) Dark brown crystals; M.p.: $262-264{ }^{\circ} \mathrm{C}$; $\mathrm{R} f$ value: 0.32; Yield: 92.45\%; IR $\left(\mathrm{KBr}, \mathrm{cm}^{-1}\right): 3086(\mathrm{C}-\mathrm{H}$ str., phenyl nucleus), 1530 ( $\mathrm{C}=\mathrm{C}$ str., phenyl nucleus), 1679 ( $\mathrm{NH}-\mathrm{C}=\mathrm{O}$ str.), 1573 ( $\mathrm{N}=\mathrm{CH}$ str., of pyrimidine), 2929 (C-H str., $\mathrm{CH}_{2}$ ), 1219 (C-NH- str.), 1348 (C-N sym. str., Ar- $\mathrm{NO}_{2}$ ); ${ }^{1} \mathrm{H}-\mathrm{NMR}\left(\delta\right.$, DMSO- $\left.d_{6}\right): 6.74-9.09(\mathrm{~m}, 20 \mathrm{H}$, $\mathrm{Ar}=\mathrm{H}-2, \mathrm{H}-3, \mathrm{H}-5, \mathrm{H}-6,\left(\mathrm{H}-2^{\prime \prime}, \mathrm{H}-3^{\prime \prime}, \mathrm{H}-5^{\prime \prime}, \mathrm{H}-6^{\prime \prime}\right) \times 2$, $\left.\left(\mathrm{H}-2^{\prime \prime \prime}, \mathrm{H}-3^{\prime \prime \prime}, \mathrm{H}-5^{\prime \prime \prime}, \mathrm{H}-6^{\prime \prime \prime}\right) \times 2\right\}, 7.04$ (s, $2 \mathrm{H}, \mathrm{CH}$ of pyrimidine), $8.01\left(\mathrm{~s}, 2 \mathrm{H},(\mathrm{NH})_{2}\right), 3.37\left(\mathrm{~s}, 4 \mathrm{H},\left(\mathrm{CH}_{2}\right)_{2}\right) ;{ }^{13} \mathrm{C}-\mathrm{NMR}$ $\left(\delta\right.$, DMSO- $\left.d_{6}\right): 127.3(\mathrm{C}-2, \mathrm{C}-3, \mathrm{C}-5, \mathrm{C}-6), 138.9$ (C-1, C-4); $164.0\left(\mathrm{C}-1^{\prime}, \mathrm{C}-3^{\prime}\right), 112\left(\mathrm{C}-2^{\prime}\right), 148.3\left(\mathrm{C}-5^{\prime}\right)$ pyrimidine; 126.3 (C-2" , C-6"), 121.5 (C-3", C-5"), $147.6\left(\mathrm{C}-4^{\prime \prime}\right)$; $152.5\left(\mathrm{C}-1^{\prime \prime \prime}\right), 115.3\left(\mathrm{C}-2^{\prime \prime \prime}, \mathrm{C}-6^{\prime \prime \prime}\right), 127.5\left(\mathrm{C}-3^{\prime \prime \prime}, \mathrm{C}-5^{\prime \prime \prime}\right)$, $135.6\left(\mathrm{C}-4^{\prime \prime \prime}\right), 168.2(\mathrm{C}=\mathrm{O}), 45.0\left(\mathrm{CH}_{2}\right)$; Elem. Anal. Calcd. $\mathrm{C}_{42} \mathrm{H}_{30} \mathrm{~N}_{12} \mathrm{O}_{10}$ : C, 58.47; H, 3.50; N, 19.48; Found: C, 58.41; $\mathrm{H}, 3.48 ; \mathrm{N}, 19.46$; MS ES + (ToF): $m / z 864\left[\mathrm{M}^{+}+1\right]$.

$N, N^{\prime}-\left(6,6^{\prime}-(1,4-P h e n y l e n e) b i s(4-(4-n i t r o p h e n y l) p y r i m-\right.$ idine-6,2-diyl))bis(2-((2,4-dinitrophenyl) amino)acetamide) (3) Brown crystals; M.p.: $257-259{ }^{\circ} \mathrm{C}$; $\mathrm{R} f$ value: 0.41; Yield: 92.67\%; IR ( $\mathrm{KBr}$ pellets, $\left.\mathrm{cm}^{-1}\right): 3211(\mathrm{C}-\mathrm{H}$ str., phenyl nucleus), 1529 ( $\mathrm{C}=\mathrm{C}$ str., phenyl nucleus), 1620 ( $\mathrm{NH}-\mathrm{C}=\mathrm{O}$ str.), 1675 ( $\mathrm{N}=\mathrm{CH}$ str., of pyrimidine), 3086 (C-H str., $\mathrm{CH}_{2}$ ), 1274 (C-NH- str.), 1345 (C-N sym. str., $\left.\mathrm{Ar}-\mathrm{NO}_{2}\right) ;{ }^{1} \mathrm{H}-\mathrm{NMR}\left(\delta, \mathrm{DMSO}-d_{6}\right): 6.95-8.90\{\mathrm{~m}, 18 \mathrm{H}$, $\mathrm{Ar}=\mathrm{H}-2, \mathrm{H}-3, \mathrm{H}-5, \mathrm{H}-6,\left(\mathrm{H}-2^{\prime \prime}, \mathrm{H}-3^{\prime \prime}, \mathrm{H}-5^{\prime \prime}, \mathrm{H}-6^{\prime \prime}\right) \times 2$, $\left.\left(\mathrm{H}-3^{\prime \prime \prime}, \mathrm{H}-5^{\prime \prime \prime}, \mathrm{H}-6^{\prime \prime \prime}\right) \times 2\right\}, 7.87\left(\mathrm{~s}, 2 \mathrm{H},\left(\mathrm{CH}_{2}\right.\right.$ of pyrimidine), $8.01\left(\mathrm{~s}, 2 \mathrm{H},(\mathrm{NH})_{2}\right), 3.40\left(\mathrm{~s}, 4 \mathrm{H},\left(\mathrm{CH}_{2}\right)_{2}\right) ;{ }^{13} \mathrm{C}-\mathrm{NMR}$ $\left(\delta\right.$, DMSO- $\left.d_{6}\right): 128.6$ (C-2, C-3, C-5, C-6), 135.05 (C-1, C-4); 164.0 (C-1', C-3'), $102\left(\mathrm{C}-2^{\prime}\right), 149.7$ (C-5') pyrimidine; $141.0\left(\mathrm{C}-1^{\prime \prime}\right), 127.3\left(\mathrm{C}-2^{\prime \prime}, \mathrm{C}-6^{\prime \prime}\right), 124.9\left(\mathrm{C}-3^{\prime \prime}, \mathrm{C}-5^{\prime \prime}\right)$, $148.6\left(\mathrm{C}-4^{\prime \prime}\right)$; $152.5\left(\mathrm{C}-1^{\prime \prime \prime}\right), 135.6\left(\mathrm{C}-2^{\prime \prime \prime}\right), 119.7\left(\mathrm{C}-3^{\prime \prime \prime}\right)$, 138.9 (C-4"'), $130.2\left(\mathrm{C}-5^{\prime \prime \prime}\right) ; 168.2(\mathrm{C}=\mathrm{O}), 51.0\left(\mathrm{CH}_{2}\right)$; Elem. Anal. Calcd. $\mathrm{C}_{42} \mathrm{H}_{28} \mathrm{~N}_{14} \mathrm{O}_{14}: \mathrm{C}, 52.95 ; \mathrm{H}, 2.96 ; \mathrm{N}$,
20.58; Found: C, 52.85; H, 2.46; N, 20.48; MS ES + (ToF): $m / z 954\left[\mathrm{M}^{+}+1\right]$.

$N, N^{\prime}-\left(6,6{ }^{\prime}-(1,4-P h e n y l e n e) b i s(4-(4-n i t r o p h e n y l) p y r i m-\right.$ idine-6,2-diyl))bis(2-((4-methoxyphenyl) amino)acetamide) (4) Brown crystals; M.p.: $215-217{ }^{\circ} \mathrm{C}$; $\mathrm{R} f$ value: 0.53; Yield: 89.44\%; IR ( $\mathrm{KBr}$ pellets, $\left.\mathrm{cm}^{-1}\right)$ : $3084(\mathrm{C}-\mathrm{H}$ str., phenyl nucleus), 1529 ( $\mathrm{C}=\mathrm{C}$ str., phenyl nucleus), $1680(\mathrm{NH}-\mathrm{C}=\mathrm{O}$ str.), $1572(\mathrm{~N}=\mathrm{CH}$ str., of pyrimidine), 1221 (C-NH- str.), 1347 (C-N sym. str., $\mathrm{Ar}-\mathrm{NO}_{2}$ ), 1088 $\left(\mathrm{C}-\mathrm{O}-\mathrm{C}\right.$ str., $\left.-\mathrm{OCH}_{3}\right) ;{ }^{1} \mathrm{H}-\mathrm{NMR}\left(\delta\right.$, DMSO- $\left.d_{6}\right)$ : 7.04$9.09\left\{\mathrm{~m}, 20 \mathrm{H}, \mathrm{Ar}=\mathrm{H}-2, \mathrm{H}-3, \mathrm{H}-5, \mathrm{H}-6,\left(\mathrm{H}-2^{\prime \prime}, \mathrm{H}-3^{\prime \prime}\right.\right.$, $\left.\left.\mathrm{H}-5^{\prime \prime}, \mathrm{H}-6^{\prime \prime}\right) \times 2,\left(\mathrm{H}-2^{\prime \prime \prime}, \mathrm{H}-3^{\prime \prime \prime}, \mathrm{H}-5^{\prime \prime \prime}, \mathrm{H}-6^{\prime \prime \prime}\right) \times 2\right\}, 7.75(\mathrm{~s}$, $2 \mathrm{H},(\mathrm{CH})_{2}$ of pyrimidine), $8.0\left(\mathrm{~s}, 2 \mathrm{H},(\mathrm{NH})_{2}\right), 3.38(\mathrm{~s}, 6 \mathrm{H}$, $\left.\left(\mathrm{OCH}_{3}\right)_{2}\right) ;{ }^{13} \mathrm{C}-\mathrm{NMR}\left(\delta\right.$, DMSO- $\left.d_{6}\right): 127.3(\mathrm{C}-2, \mathrm{C}-3, \mathrm{C}-5$, C-6), 138.5 (C-1, C-4); 164.0 (C-1', C-3'), 108 (C-2'), 148.7 (C-5') pyrimidine; $142.0\left(\mathrm{C}-1^{\prime \prime}\right), 127.5\left(\mathrm{C}-2^{\prime \prime}, \mathrm{C}-6^{\prime \prime}\right), 124.9$ (C-3", C-5"), $149.6\left(\mathrm{C}-4^{\prime \prime}\right)$; 138.5 (C-1"'), $115.3\left(\mathrm{C}-2^{\prime \prime \prime}\right.$, C-6"'), $115.0\left(\mathrm{C}-3^{\prime \prime \prime}, \mathrm{C}-5^{\prime \prime \prime}\right), 171.2(\mathrm{C}=\mathrm{O}), 51.0\left(\mathrm{CH}_{2}\right), 53.0$ $\left(p-\mathrm{OCH}_{3}\right)$; Elem. Anal. Calcd. $\mathrm{C}_{44} \mathrm{H}_{36} \mathrm{~N}_{10} \mathrm{O}_{8}$ : C, 63.46; H, 4.36; N, 16.82; Found: C, 63.44; H, 4.37; N, 16.85; MS $\mathrm{ES}+(\mathrm{ToF}): m / z 834\left[\mathrm{M}^{+}+1\right]$.

$N, N^{\prime}-\left(6,6^{\prime}-(1,4-P h e n y l e n e) b i s(4-(4-n i t r o p h e n y l) p y r i m i-\right.$ dine-6,2-diyl))bis(2-(p-tolylamino) acetamide) (5) Brown crystals; M.p.: $250-252^{\circ} \mathrm{C}$; $R$ f value: 0.30; Yield: $85.43 \%$; IR ( $\mathrm{KBr}$ pellets, $\mathrm{cm}^{-1}$ ): 3084 (C-H str., phenyl nucleus), 1529 ( $\mathrm{C}=\mathrm{C}$ str., phenyl nucleus), $1681(\mathrm{NH}-\mathrm{C}=\mathrm{O}$ str.), 1569 $\left(\mathrm{N}=\mathrm{CH}\right.$ str., of pyrimidine), $2866\left(\mathrm{C}-\mathrm{H}\right.$ str., $\left.\mathrm{CH}_{2}\right), 1217$ (C-NH- str.), 1348 (C-N sym. str., $\left.\mathrm{Ar}-\mathrm{NO}_{2}\right) ;{ }^{1} \mathrm{H}-\mathrm{NMR}$ $\left(\delta\right.$, DMSO- $\left.d_{6}\right): 6.99-8.56\{\mathrm{~m}, 20 \mathrm{H}, \mathrm{Ar}=\mathrm{H}-2, \mathrm{H}-3, \mathrm{H}-5$, H-6, (H-2" $\left., \mathrm{H}-3^{\prime \prime}, \mathrm{H}-5^{\prime \prime}, \mathrm{H}-6^{\prime \prime}\right) \times 2,\left(\mathrm{H}-2^{\prime \prime \prime}, \mathrm{H}-3^{\prime \prime \prime}, \mathrm{H}-5^{\prime \prime \prime}\right.$, $\left.\left.\mathrm{H}-6^{\prime \prime \prime}\right) \times 2\right\}, 7.48\left(\mathrm{~s}, 2 \mathrm{H},(\mathrm{CH})_{2}\right.$ of pyrimidine), 8.03 (s, $\left.2 \mathrm{H},(\mathrm{NH})_{2}\right), 2.51\left(\mathrm{~s}, 6 \mathrm{H},\left(\mathrm{CH}_{3}\right)_{2}\right) ;{ }^{13} \mathrm{C}-\mathrm{NMR}(\delta$, DMSO$\left.d_{6}\right): 128.8,128.4(\mathrm{C}-2, \mathrm{C}-3, \mathrm{C}-5, \mathrm{C}-6), 136.8,(\mathrm{C}-1, \mathrm{C}-4)$; $168.0\left(\mathrm{C}-1^{\prime}, \mathrm{C}-3^{\prime}\right), 108\left(\mathrm{C}-2^{\prime}\right), 149.7\left(\mathrm{C}-5^{\prime}\right)$ pyrimidine; $141.0\left(\mathrm{C}-1^{\prime \prime}\right), 126.4\left(\mathrm{C}-2^{\prime \prime}, \mathrm{C}-6^{\prime \prime}\right), 123.8$ (C-3") C-5"), 149.7 (C-4"); 144.5 (C-1"'), 117.0 (C-2"', C-6"'), 129.6 (C-3"', C-5"' $), 188.0(\mathrm{C}=\mathrm{O}), 48.0\left(\mathrm{CH}_{2}\right), 20.5\left(p-\mathrm{CH}_{3}\right)$; Elem. Anal. Calcd. $\mathrm{C}_{44} \mathrm{H}_{36} \mathrm{~N}_{10} \mathrm{O}_{6}$ : C, 65.99; H, 4.53; N, 17.49; Found: C, 65.92; H, 4.49; N, 17.40; MS ES + (ToF): $m / z 802\left[\mathrm{M}^{+}+1\right]$.

$N, N^{\prime}-\left(6,6^{\prime}-(1,4-P h e n y l e n e) b i s(4-(4-n i t r o p h e n y l) p y r i m i-\right.$ dine-6,2-diyl))bis(2-((4-chlorophenyl) amino)acetamide) (6) Brown crystals; M.p.: $168-170{ }^{\circ} \mathrm{C}$; $\mathrm{R} f$ value: 0.42 ; Yield: $87.58 \%$; IR (KBr pellets, $\left.\mathrm{cm}^{-1}\right)$ : 3084 (C-H str., phenyl nucleus), 1530 ( $\mathrm{C}=\mathrm{C}$ str., phenyl nucleus), 1687 (NH$\mathrm{C}=\mathrm{O}$ str.), 1603 ( $\mathrm{N}=\mathrm{CH}$ str., of pyrimidine), $2866(\mathrm{C}-\mathrm{H}$ str., $\left.\mathrm{CH}_{2}\right), 1215$ (C-NH- str.), 1348 (C-N sym. str., Ar$\left.\mathrm{NO}_{2}\right), 739$ (C-Cl str., Ar-Cl); ${ }^{1} \mathrm{H}-\mathrm{NMR}\left(\delta\right.$, DMSO- $\left.d_{6}\right)$ : 6.35-9.08 $\left\{\mathrm{m}, 20 \mathrm{H}, \mathrm{Ar}=\mathrm{H}-2, \mathrm{H}-3, \mathrm{H}-5, \mathrm{H}-6,\left(\mathrm{H}-2^{\prime \prime}, \mathrm{H}-3^{\prime \prime}\right.\right.$, $\left.\left.\mathrm{H}-5^{\prime \prime}, \mathrm{H}-6^{\prime \prime}\right) \times 2,\left(\mathrm{H}-2^{\prime \prime \prime}, \mathrm{H}-3^{\prime \prime \prime}, \mathrm{H}-5^{\prime \prime \prime}, \mathrm{H}-6^{\prime \prime \prime}\right) \times 2\right\}, 7.49(\mathrm{~s}$, 
$2 \mathrm{H},(\mathrm{CH})_{2}$ of pyrimidine), $8.00\left(\mathrm{~s}, 2 \mathrm{H},(\mathrm{NH})_{2}\right), 3.36(\mathrm{~s}, 4 \mathrm{H}$, $\left.\left(\mathrm{CH}_{2}\right)_{2}\right) ;{ }^{13} \mathrm{C}-\mathrm{NMR}\left(\delta\right.$, DMSO- $\left.d_{6}\right): 128.4(\mathrm{C}-2, \mathrm{C}-3, \mathrm{C}-5$,

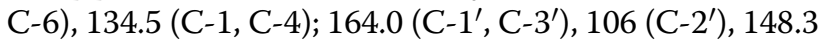
$\left(\mathrm{C}-5^{\prime}\right)$ pyrimidine; $127.4\left(\mathrm{C}-2^{\prime \prime}, \mathrm{C}-6^{\prime \prime}\right), 124.9\left(\mathrm{C}-3^{\prime \prime}, \mathrm{C}-5^{\prime \prime}\right)$, $147.6\left(\mathrm{C}-4^{\prime \prime}\right) ; 145.5\left(\mathrm{C}-1^{\prime \prime \prime}\right), 115.1$ (C-2"', C-6"'), 129.9 (C-3"', C-5"'), $126.1\left(\mathrm{C}-4^{\prime \prime \prime}\right) ; 165.2(\mathrm{C}=\mathrm{O}), 53.0\left(\mathrm{CH}_{2}\right)$; Elem. Anal. Calcd. $\mathrm{C}_{42} \mathrm{H}_{30} \mathrm{Cl}_{2} \mathrm{~N}_{10} \mathrm{O}_{6}$ : C, 59.94; H, 3.59; N, 16.64; Found: C, 59.90; H, 3.51; N, 16.60; MS ES + (ToF): $m / z 843\left[\mathrm{M}^{+}+1\right]$.

$N, N^{\prime}-\left(6,6^{\prime}-(1,4-P h e n y l e n e) b i s(4-(4-n i t r o p h e n y l)\right.$ pyrimidine-6,2-diyl))bis(2-((2-chloro-4-nitro phenyl)amino) acetamide) (7) Brown yellow crystals; M.p.: $170-172{ }^{\circ} \mathrm{C}$; $\mathrm{R} f$ value: 0.60; Yield: $87.12 \%$; IR ( $\mathrm{KBr}$ pellets, $\mathrm{cm}^{-1}$ ): 3085 (C-H str., phenyl nucleus), 1530 ( $\mathrm{C}=\mathrm{C}$ str., phenyl nucleus), 1688 ( $\mathrm{NH}-\mathrm{C}=\mathrm{O}$ str.), 1603 ( $\mathrm{N}=\mathrm{CH}$ str., of pyrimidine), 2869 (C-H str., $\mathrm{CH}_{2}$ ), 1215 (C-NH- str.), 1348 (C-N sym. str., $\left.\mathrm{Ar}-\mathrm{NO}_{2}\right), 740(\mathrm{C}-\mathrm{Cl}$ str., $\mathrm{Ar}-\mathrm{Cl}) ;{ }^{1} \mathrm{H}-\mathrm{NMR}(\delta$, DMSO$\left.d_{6}\right): 6.48-9.08\left\{\mathrm{~m}, 18 \mathrm{H}, \mathrm{Ar}=\mathrm{H}-2, \mathrm{H}-3, \mathrm{H}-5, \mathrm{H}-6,\left(\mathrm{H}-2^{\prime \prime}\right.\right.$, $\left.\left.\mathrm{H}-3^{\prime \prime}, \mathrm{H}-5^{\prime \prime}, \mathrm{H}-6^{\prime \prime}\right) \times 2,\left(\mathrm{H}-3^{\prime \prime \prime}, \mathrm{H}-5^{\prime \prime \prime}, \mathrm{H}-6^{\prime \prime \prime}\right) \times 2\right\}, 7.87(\mathrm{~s}$, $2 \mathrm{H},(\mathrm{CH})_{2}$ of pyrimidine), $8.01\left(\mathrm{~s}, 2 \mathrm{H},(\mathrm{NH})_{2}\right), 3.37(\mathrm{~s}, 4 \mathrm{H}$, $\left.\left(\mathrm{CH}_{2}\right)_{2}\right) ;{ }^{13} \mathrm{C}-\mathrm{NMR}\left(\delta\right.$, DMSO- $\left.d_{6}\right): 129.3(\mathrm{C}-2, \mathrm{C}-3, \mathrm{C}-5$, C-6), 135.8 (C-1, C-4); 163.0 (C-1', C-3'), 113 (C-2'), 148.7 (C-5') pyrimidine; $126.3\left(\mathrm{C}-2^{\prime \prime}, \mathrm{C}-6^{\prime \prime}\right), 124.9\left(\mathrm{C}-3^{\prime \prime}, \mathrm{C}-5^{\prime \prime}\right)$, $149.3\left(\mathrm{C}-4^{\prime \prime}\right) ; 151.3\left(\mathrm{C}-1^{\prime \prime \prime}\right), 124.3\left(\mathrm{C}-2^{\prime \prime \prime}\right), 138.9\left(\mathrm{C}-4^{\prime \prime \prime}\right), 125.1$ (C-3"'), $122.1\left(\mathrm{C}-5^{\prime \prime \prime}\right)$; $166.2(\mathrm{C}=\mathrm{O}), 51.0\left(\mathrm{CH}_{2}\right)$; Elem. Anal. Calcd. $\mathrm{C}_{42} \mathrm{H}_{28} \mathrm{Cl}_{2} \mathrm{~N}_{12} \mathrm{O}_{10}$ : C, 54.15; H, 3.03; N, 18.04; Found: C, 54.11; H, 3.00; N, 18.12; MS ES + (ToF): $m / z 933\left[\mathrm{M}^{+}\right.$ $+1]$.

$N, N^{\prime}-\left(6,6^{\prime}-(1,4-P h e n y l e n e) b i s(4-(4-n i t r o p h e n y l) p y r i m i-\right.$ dine-6,2-diyl))bis(2-((2-nitrophenyl) amino)acetamide) (8) Light Brown crystals; M.p.: $163-165{ }^{\circ} \mathrm{C} ; \mathrm{R} f$ value: 0.34; Yield: 84.23\%; IR ( $\mathrm{KBr}$ pellets, $\left.\mathrm{cm}^{-1}\right): 3085(\mathrm{C}-\mathrm{H}$ str., phenyl nucleus), 1530 ( $\mathrm{C}=\mathrm{C}$ str., phenyl nucleus), $1688(\mathrm{NH}-\mathrm{C}=\mathrm{O}$ str.), 1603 ( $\mathrm{N}=\mathrm{CH}$ str., of pyrimidine), 2868 (C-H str., $\left.\mathrm{CH}_{2}\right), 1215$ (C-NH- str.), 1348 (C-N sym. str., $\left.\mathrm{Ar}-\mathrm{NO}_{2}\right) ;{ }^{1} \mathrm{H}-\mathrm{NMR}\left(\delta\right.$, DMSO- $\left.d_{6}\right)$ : 7.03-8.25 (m, 20H, Ar $=\mathrm{H}-2, \mathrm{H}-3, \mathrm{H}-5, \mathrm{H}-6,\left(\mathrm{H}-2^{\prime \prime}, \mathrm{H}-3^{\prime \prime}, \mathrm{H}-5^{\prime \prime}\right.$, $\left.\left.\mathrm{H}-6^{\prime \prime}\right) \times 2,\left(\mathrm{H}-3^{\prime \prime \prime}, \mathrm{H}-4^{\prime \prime \prime}, \mathrm{H}-5^{\prime \prime \prime}, \mathrm{H}-6^{\prime \prime \prime}\right) \times 2\right\}, 7.79(\mathrm{~s}, 2 \mathrm{H}$, $(\mathrm{CH})_{2}$ of pyrimidine), $8.04\left(\mathrm{~s}, 2 \mathrm{H},(\mathrm{NH})_{2}\right), 3.38(\mathrm{~s}, 4 \mathrm{H}$, $\left.\left(\mathrm{CH}_{2}\right)_{2}\right) ;{ }^{13} \mathrm{C}-\mathrm{NMR}\left(\delta\right.$, DMSO- $\left.d_{6}\right): 127.4(\mathrm{C}-2, \mathrm{C}-3, \mathrm{C}-5$, C-6), 135.6 (C-1, C-4); 162.0 (C-1', C-3'), 105 (C-2'), 148.7 $\left(\mathrm{C}-5^{\prime}\right)$ pyrimidine; $141.0\left(\mathrm{C}-1^{\prime \prime}\right), 126.5\left(\mathrm{C}-2^{\prime \prime}, \mathrm{C}-6^{\prime \prime}\right), 124.3$ (C-3", C-5"), $149.6\left(\mathrm{C}-4^{\prime \prime}\right) ; 147.5\left(\mathrm{C}-1^{\prime \prime \prime}\right), 130.1\left(\mathrm{C}-2^{\prime \prime \prime}\right)$, 125.9 (C-3"'), 119.1 (C-4"'), $136.2\left(\mathrm{C}-5^{\prime \prime \prime}\right) ; 164.2(\mathrm{C}=\mathrm{O})$, $50.0\left(\mathrm{CH}_{2}\right)$; Elem. Anal. Calcd. $\mathrm{C}_{42} \mathrm{H}_{30} \mathrm{~N}_{12} \mathrm{O}_{10}$ : C, 58.47; H, 3.50; N, 19.48; Found: C, 58.42; H, 3.45; N, 19.41; MS $\mathrm{ES}+(\mathrm{ToF}): m / z 864\left[\mathrm{M}^{+}+1\right]$.

$N, N^{\prime}-\left(6,6^{\prime}-(1,4-P h e n y l e n e) b i s(4-(4-n i t r o p h e n y l) p y r i m-\right.$ idine-6,2-diyl))bis(2-(2-chlorophenyl) acetamide)

(9) Canary yellow crystals; M.p.: $150-152{ }^{\circ} \mathrm{C}$; $\mathrm{R} f$ value:
0.58; Yield: 75.25\%; IR ( $\mathrm{KBr}$ pellets, $\mathrm{cm}^{-1}$ ): 3075 (C-H str., phenyl nucleus), 1521 ( $\mathrm{C}=\mathrm{C}$ str., phenyl nucleus), 1690 ( $\mathrm{NH}-\mathrm{C}=\mathrm{O}$ str.), 1598 ( $\mathrm{N}=\mathrm{CH}$ str., of pyrimidine), 2851 (C-H str., $\left.\mathrm{CH}_{2}\right), 1210$ (C-NH- str.), 1344 (C-N sym. str., $\left.\mathrm{Ar}-\mathrm{NO}_{2}\right), 757(\mathrm{C}-\mathrm{Cl}$ str., $\mathrm{Ar}-\mathrm{Cl}) ;{ }^{1} \mathrm{H}-\mathrm{NMR}(\delta$, DMSO$\left.d_{6}\right): 7.80-8.25\left\{\mathrm{~m}, 20 \mathrm{H}, \mathrm{Ar}=\mathrm{H}-2, \mathrm{H}-3, \mathrm{H}-5, \mathrm{H}-6,\left(\mathrm{H}-2^{\prime \prime}\right.\right.$, $\left.\left.\mathrm{H}-3^{\prime \prime}, \mathrm{H}-5^{\prime \prime}, \mathrm{H}-6^{\prime \prime}\right) \times 2,\left(\mathrm{H}-3^{\prime \prime \prime}, \mathrm{H}-4^{\prime \prime \prime}, \mathrm{H}-5^{\prime \prime \prime}, \mathrm{H}-6^{\prime \prime \prime}\right) \times 2\right\}$, $7.80\left(\mathrm{~s}, 2 \mathrm{H},(\mathrm{CH})_{2}\right.$ of pyrimidine), $8.00\left(\mathrm{~s}, 2 \mathrm{H},(\mathrm{NH})_{2}\right)$, $3.37\left(\mathrm{~s}, 4 \mathrm{H},\left(\mathrm{CH}_{2}\right)_{2}\right) ;{ }^{13} \mathrm{C}-\mathrm{NMR}\left(\delta\right.$, DMSO- $\left.d_{6}\right): 128.6(\mathrm{C}-2$,

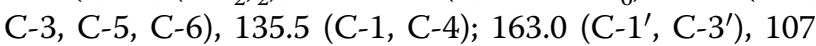
(C-2'), $149.7\left(\mathrm{C}-5^{\prime}\right)$ pyrimidine; $141.0\left(\mathrm{C}-1^{\prime \prime}\right), 127.5\left(\mathrm{C}-2^{\prime \prime}\right.$, C-6"), $123.5\left(\mathrm{C}-3^{\prime \prime}, \mathrm{C}-5^{\prime \prime}\right), 149.8\left(\mathrm{C}-4^{\prime \prime}\right) ; 134.5\left(\mathrm{C}-1^{\prime \prime \prime}\right), 129.1$ (C-2"', C-3"', C-4"' $), 127.9$ (C-5"'), $133.1\left(\mathrm{C}-6^{\prime \prime \prime}\right) ; 156.2$ $(\mathrm{C}=\mathrm{O}), 39\left(\mathrm{CH}_{2}\right)$; Elem. Anal. Calcd. $\mathrm{C}_{42} \mathrm{H}_{30} \mathrm{Cl}_{2} \mathrm{~N}_{10} \mathrm{O}_{6}$ : C, 59.94; H, 3.59; N, 16.64; Found: C, 59.90; H, 3.53; N, 16.61; MS ES + (ToF): $m / z 843\left[\mathrm{M}^{+}+1\right]$.

$N, N^{\prime}-\left(6,6^{\prime}-(1,4-P h e n y l e n e) b i s(4-(4-n i t r o p h e n y l) p y r i m-\right.$ idine-6,2-diyl))bis(2-(2-chlorophenyl) acetamide) (10) Light yellow crystals; M.p.: $138-140^{\circ} \mathrm{C}$; R fvalue: 0.31 ; Yield: $80.22 \%$; IR ( $\mathrm{KBr}$ pellets, $\mathrm{cm}^{-1}$ ): 3074 (C-H str., phenyl nucleus), 1521 ( $\mathrm{C}=\mathrm{C}$ str., phenyl nucleus), $1690(\mathrm{NH}-\mathrm{C}=\mathrm{O}$ str.), 1597 ( $\mathrm{N}=\mathrm{CH}$ str., of pyrimidine), 2854 ( $\mathrm{C}-\mathrm{H}$ str., $\mathrm{CH}_{2}$ ), 1210 (C-NH- str.), 1344 (C-N sym. str., Ar- $\mathrm{NO}_{2}$ ), 757 $(\mathrm{C}-\mathrm{Cl}$ str., $\mathrm{Ar}-\mathrm{Cl}) ;{ }^{1} \mathrm{H}-\mathrm{NMR}\left(\delta, \mathrm{DMSO}-d_{6}\right): 7.82\{\mathrm{~m}, 20 \mathrm{H}$, $\mathrm{Ar}=\mathrm{H}-2, \mathrm{H}-3, \mathrm{H}-5, \mathrm{H}-6,\left(\mathrm{H}-2^{\prime \prime}, \mathrm{H}-3^{\prime \prime}, \mathrm{H}-5^{\prime \prime}, \mathrm{H}-6^{\prime \prime}\right) \times 2$, $\left.\left(\mathrm{H}-2^{\prime \prime \prime}, \mathrm{H}-4^{\prime \prime \prime}, \mathrm{H}-5^{\prime \prime \prime}, \mathrm{H}-6^{\prime \prime \prime}\right) \times 2\right\}, 7.80\left(\mathrm{~s}, 2 \mathrm{H},(\mathrm{CH})_{2}\right.$ of pyrimidine), $8.00\left(\mathrm{~s}, 2 \mathrm{H},(\mathrm{NH})_{2}\right), 3.38\left(\mathrm{~s}, 4 \mathrm{H},\left(\mathrm{CH}_{2}\right)_{2}\right) ;{ }^{13} \mathrm{C}-\mathrm{NMR}$ $\left(\delta\right.$, DMSO- $\left.d_{6}\right)$ : 128.3 (C-2, C-3, C-5, C-6), 135.5 (C-1, C-4); $165.0\left(\mathrm{C}-1^{\prime}, \mathrm{C}-3^{\prime}\right), 108\left(\mathrm{C}-2^{\prime}\right), 149.8\left(\mathrm{C}-5^{\prime}\right)$ pyrimidine; 142.0 $\left(\mathrm{C}-1^{\prime \prime}\right), 127.5\left(\mathrm{C}-2^{\prime \prime}, \mathrm{C}-6^{\prime \prime}\right), 123.5\left(\mathrm{C}-3^{\prime \prime}, \mathrm{C}-5^{\prime \prime}\right), 149.6\left(\mathrm{C}-4^{\prime \prime}\right)$; 149.5 (C-1"' ), 110 (C-2'"'), 134.9 (C-3"'), $120.4\left(\mathrm{C}-5^{\prime \prime \prime}\right) ; 163.2$ $(\mathrm{C}=\mathrm{O}), 56.0\left(\mathrm{CH}_{2}\right)$; Elem. Anal. Calcd. $\mathrm{C}_{42} \mathrm{H}_{30} \mathrm{Cl}_{2} \mathrm{~N}_{10} \mathrm{O}_{6}$ : C, 59.94; H, 3.59; N, 16.64; Found: C, 59.90; H, 3.52; N, 16.67; MS ES + (ToF): $m / z 843\left[\mathrm{M}^{+}+1\right]$.

$N, N^{\prime}-(6,6$ - $-(1,4-$ Phenylene)bis(4-(4-nitrophenyl)pyrimidine-6,2-diyl))bis(2-((4-fluorophenyl) amino)acetamide) (11) Yellow crystals; M.p.: $123-125{ }^{\circ} \mathrm{C}$; $\mathrm{R} f$ value: 0.33; Yield: 85.63\%; IR ( $\mathrm{KBr}$ pellets, $\left.\mathrm{cm}^{-1}\right)$ : $3073(\mathrm{C}-\mathrm{H}$ str., phenyl nucleus), 1518 ( $\mathrm{C}=\mathrm{C}$ str., phenyl nucleus), 1665 $(\mathrm{NH}-\mathrm{C}=\mathrm{O}$ str.), $1596(\mathrm{~N}=\mathrm{CH}$ str., of pyrimidine), 2852 (C-H str., $\mathrm{CH}_{2}$ ), 1210 (C-NH- str.), 1338 (C-N sym. str., $\left.\mathrm{Ar}-\mathrm{NO}_{2}\right), 1105$ (C-F str., Ar-F); ${ }^{1} \mathrm{H}-\mathrm{NMR}\left(\delta\right.$, DMSO- $\left.d_{6}\right)$ : 7.25-8.69 $\left\{\mathrm{m}, 20 \mathrm{H}, \mathrm{Ar}=\mathrm{H}-2, \mathrm{H}-3, \mathrm{H}-5, \mathrm{H}-6,\left(\mathrm{H}-2^{\prime \prime}, \mathrm{H}-3^{\prime \prime}\right.\right.$, $\left.\left.\mathrm{H}-5^{\prime \prime}, \mathrm{H}-6^{\prime \prime}\right) \times 2,\left(\mathrm{H}-2^{\prime \prime \prime}, \mathrm{H}-3^{\prime \prime \prime}, \mathrm{H}-5^{\prime \prime \prime}, \mathrm{H}-6^{\prime \prime \prime}\right) \times 2\right\}, 7.39(\mathrm{~s}$, $2 \mathrm{H},(\mathrm{CH})_{2}$ of pyrimidine), $8.01\left(\mathrm{~s}, 2 \mathrm{H},(\mathrm{NH})_{2}\right), 3.34(\mathrm{~s}, 4 \mathrm{H}$, $\left.\left(\mathrm{CH}_{2}\right)_{2}\right) ;{ }^{13} \mathrm{C}-\mathrm{NMR}\left(\delta\right.$, DMSO- $\left.d_{6}\right): 129.3(\mathrm{C}-2, \mathrm{C}-3, \mathrm{C}-5$, C-6), 136.7 (C-1, C-4); 161.0 (C-1', C-3'), 105 (C-2'), 149.8 $\left(\mathrm{C}-5^{\prime}\right)$ pyrimidine; $142.0\left(\mathrm{C}-1^{\prime \prime}\right), 126.4\left(\mathrm{C}-2^{\prime \prime}, \mathrm{C}-6^{\prime \prime}\right), 124.5$ $\left(\mathrm{C}-3^{\prime \prime}, \mathrm{C}-5^{\prime \prime}\right), 147.4\left(\mathrm{C}-4^{\prime \prime}\right) ; 144.5\left(\mathrm{C}-1^{\prime \prime \prime}\right), 115.9\left(\mathrm{C}-3^{\prime \prime \prime}\right.$, C-5"'), 119.0 (C-2'", C-6"'), 159.8 (C-5"'); $169.2(\mathrm{C}=\mathrm{O})$, $68.0\left(\mathrm{CH}_{2}\right)$; Elem. Anal. Calcd. $\mathrm{C}_{42} \mathrm{H}_{30} \mathrm{~F}_{2} \mathrm{~N}_{10} \mathrm{O}_{6}$ : C, 62.37; 
H, 3.74; N, 17.32; Found: C, 62.33; H, 3.72; N, 17.35; MS $\mathrm{ES}+(\mathrm{ToF}): m / z 810\left[\mathrm{M}^{+}+1\right]$.

$N, N^{\prime}-\left(6,6^{\prime}-(1,4-P h e n y l e n e) b i s(4-(4-n i t r o p h e n y l) p y r i m i-\right.$ dine-6,2-diyl))bis(2-((2-fluorophenyl) amino)acetamide) (12) Gold Yellow crystals; M.p.: $170-172{ }^{\circ} \mathrm{C}$; $\mathrm{R} f$ value: 0.25; Yield: $77.12 \%$; IR ( $\mathrm{KBr}$ pellets, $\left.\mathrm{cm}^{-1}\right)$ : $3108(\mathrm{C}-\mathrm{H}$ str., phenyl nucleus), 1519 ( $\mathrm{C}=\mathrm{C}$ str., phenyl nucleus), 1665 ( $\mathrm{NH}-\mathrm{C}=\mathrm{O}$ str.), 1600 ( $\mathrm{N}=\mathrm{CH}$ str., of pyrimidine), 2934 (C-H str., $\mathrm{CH}_{2}$ ), 1211 (C-NH- str.), 1340 (C-N sym. str., Ar- $\left.\mathrm{NO}_{2}\right), 1106$ (C-F str., Ar-F); ${ }^{1} \mathrm{H}-\mathrm{NMR}\left(\delta\right.$, DMSO- $\left.d_{6}\right)$ : 7.91-839 $\left\{\mathrm{m}, 20 \mathrm{H}, \mathrm{Ar}=\mathrm{H}-2, \mathrm{H}-3, \mathrm{H}-5, \mathrm{H}-6,\left(\mathrm{H}-2^{\prime \prime}, \mathrm{H}-3^{\prime \prime}\right.\right.$, H-5", H-6"') $\times 2$, $\left.\left(\mathrm{H}-3^{\prime \prime \prime}, \mathrm{H}-4^{\prime \prime \prime}, \mathrm{H}-5^{\prime \prime \prime}, \mathrm{H}-6^{\prime \prime \prime}\right) \times 2\right\}, 7.90(\mathrm{~s}$, $2 \mathrm{H},(\mathrm{CH})_{2}$ of pyrimidine $), 3.38\left(\mathrm{~s}, 4 \mathrm{H},\left(\mathrm{CH}_{2}\right), 8.00(\mathrm{~s}, 2 \mathrm{H}\right.$, $\left.(\mathrm{NH})_{2}\right) ;{ }^{13} \mathrm{C}-\mathrm{NMR}\left(\delta\right.$, DMSO- $\left.d_{6}\right): 129.3(\mathrm{C}-2, \mathrm{C}-3, \mathrm{C}-5$, C-6), 135.5 (C-1, C-4); 162.0 (C-1', C-3'), 105 (C-2'), 149.7 $\left(\mathrm{C}-5^{\prime}\right)$ pyrimidine; $127.5\left(\mathrm{C}-2^{\prime \prime}, \mathrm{C}-6^{\prime \prime}\right), 124.7\left(\mathrm{C}-3^{\prime \prime}, \mathrm{C}-5^{\prime \prime}\right)$, 147.6 (C-4"'); 130.5 (C-1"'), 153.2 (C-3"'), 116.9 (C-3"'), $121.9\left(\mathrm{C}-4^{\prime \prime \prime}\right) ; 188.2(\mathrm{C}=\mathrm{O}), 49.0\left(\mathrm{CH}_{2}\right)$; Elem. Anal. Calcd. $\mathrm{C}_{42} \mathrm{H}_{30} \mathrm{~F}_{2} \mathrm{~N}_{10} \mathrm{O}_{6}$ : C, 62.37; H, 3.74; N, 17.32; Found; C, 62.34; H, 3.67; N, 17.28; MS ES + (ToF): $m / z 810\left[\mathrm{M}^{+}+1\right]$.

$N, N^{\prime}-\left(6,6^{\prime}-(1,4-P h e n y l e n e) b i s(4-(4-n i t r o p h e n y l)\right.$ pyrimidine-6,2-diyl))bis(2-((4-chloro-2-nitro phenyl)amino) acetamide) (13) Light yellow crystals; M.p.: $175-177^{\circ} \mathrm{C}$; $\mathrm{R} f$ value: 0.16 ; Yield: $85.25 \%$; IR ( $\mathrm{KBr}$ pellets, $\mathrm{cm}^{-1}$ ): 3107 (C-H str., phenyl nucleus), 1519 ( $\mathrm{C}=\mathrm{C}$ str., phenyl nucleus), 1665 ( $\mathrm{NH}-\mathrm{C}=\mathrm{O}$ str.), $1600(\mathrm{~N}=\mathrm{CH}$ str., of pyrimidine), 2936 (C-H str., $\left.\mathrm{CH}_{2}\right), 1247$ (C-NH- str.), 1341 (C-N sym. str., Ar- $\left.\mathrm{NO}_{2}\right), 761(\mathrm{C}-\mathrm{Cl}$ str., $\mathrm{Ar}-\mathrm{Cl})$; ${ }^{1} \mathrm{H}-\mathrm{NMR}\left(\delta\right.$, DMSO- $\left.d_{6}\right): 7.05-8.39\{\mathrm{~m}, 18 \mathrm{H}, \mathrm{Ar}=\mathrm{H}-2$, H-3, H-5, H-6, (H-2" , H-3" , H-5", H-6") $\times 2$, (H-3"', H-5"', $\left.\left.\mathrm{H}-6^{\prime \prime \prime}\right) \times 2\right\}, 7.48\left(\mathrm{~s}, 2 \mathrm{H},(\mathrm{CH})_{2}\right.$ of pyrimidine $), 8.00(\mathrm{~s}, 2 \mathrm{H}$, $\left.(\mathrm{NH})_{2}\right), 3.66\left(\mathrm{~s}, 4 \mathrm{H},\left(\mathrm{CH}_{2}\right)_{2}\right) ;{ }^{13} \mathrm{C}-\mathrm{NMR}\left(\delta\right.$, DMSO- $\left.d_{6}\right)$ : 128.9 (C-2, C-3, C-5, C-6), 135.5 (C-1,C-4); 165.0 (C-1', C-3'), $110\left(\mathrm{C}-2^{\prime}\right), 149.8\left(\mathrm{C}-5^{\prime}\right)$ pyrimidine; $141.0\left(\mathrm{C}-1^{\prime \prime}\right)$, 126.4 (C-2", C-6"), 124.0 (C-3", C-5"), $149.9\left(\mathrm{C}-4^{\prime \prime}\right) ; 143.5$ (C-1"'), 132.2 (C-2"'), 126.5 (C-3"' ), 122.9 (C-4"'), 134.1 (C-5"'), $118.1\left(\mathrm{C}-6^{\prime \prime \prime}\right) ; 188.2(\mathrm{C}=\mathrm{O}), 52.5\left(\mathrm{CH}_{2}\right)$; Elem. Anal. Calcd. $\mathrm{C}_{42} \mathrm{H}_{28} \mathrm{Cl}_{2} \mathrm{~N}_{12} \mathrm{O}_{10}$ : C, 54.15; H, 3.03; N, 18.04; Found: C, 54.13; H, 3.00; N, 18.01; MS ES + (ToF): $m / z$ $933\left[\mathrm{M}^{+}+1\right]$.

$N, N^{\prime}-(6,6$ - $-(1,4-P h e n y l e n e) b i s(4-(4-n i t r o p h e n y l)$ pyrimidine-6,2-diyl))bis(2-((2,6-dimethyl phenyl)amino)acetamide) (14) Gold yellow crystals; M.p.: $158-160{ }^{\circ} \mathrm{C}$; $\mathrm{R} f$ value: 0.38 ; Yield: $82.33 \%$; IR (KBr pellets, $\mathrm{cm}^{-1}$ ): 3074 ( $\mathrm{C}-\mathrm{H}$ str., phenyl nucleus), 1520 ( $\mathrm{C}=\mathrm{C}$ str., phenyl nucleus), 1692 ( $\mathrm{NH}-\mathrm{C}=\mathrm{O}$ str.), 1601 ( $\mathrm{N}=\mathrm{CH}$ str., of pyrimidine), 2853 (C-H str., $\left.\mathrm{CH}_{2}\right), 1211$ (C-NH- str.), $1343\left(\mathrm{C}-\mathrm{N}\right.$ sym. str., $\left.\mathrm{Ar}-\mathrm{NO}_{2}\right){ }^{1} \mathrm{H}-\mathrm{NMR}\left(\delta, \mathrm{DMSO}-d_{6}\right)$ : 7.94-8.39 \{m, 18H, Ar $=\mathrm{H}-2, \mathrm{H}-3, \mathrm{H}-5, \mathrm{H}-6,\left(\mathrm{H}-2^{\prime \prime}, \mathrm{H}-3^{\prime \prime}\right.$, $\left.\left.\mathrm{H}-5^{\prime \prime}, \mathrm{H}-6^{\prime \prime}\right) \times 2,\left(\mathrm{H}-3^{\prime \prime \prime}, \mathrm{H}-4^{\prime \prime \prime}, \mathrm{H}-5^{\prime \prime \prime}\right) \times 2\right\}, 7.91(\mathrm{~s}, 2 \mathrm{H}$,
$(\mathrm{CH})_{2}$ of pyrimidine), $8.01\left(\mathrm{~s}, 2 \mathrm{H},(\mathrm{NH})_{2}\right), 3.39(\mathrm{~s}, 4 \mathrm{H}$, $\left.\left(\mathrm{CH}_{2}\right)_{2}\right), 2.09$ (s, 6H, $\left.\left(\mathrm{CH}_{3}\right)_{2}\right) ;{ }^{13} \mathrm{C}-\mathrm{NMR}\left(\delta\right.$, DMSO- $\left.d_{6}\right)$ : 128.6 (C-2, C-3, C-5, C-6), 135.5 (C-1, C-4); 162.0 (C-1', C-3'), $108\left(\mathrm{C}-2^{\prime}\right), 149.8\left(\mathrm{C}-5^{\prime}\right)$ pyrimidine; $141.0\left(\mathrm{C}-1^{\prime \prime}\right)$, 126.5 (C-2", C-6"), 123.5 (C-3", C-5"), 149.0 (C-4"); 145.5 (C-1"'), 119.0 (C-4"'), 127.2 (C-2"', C-6"' $), 127.9$ (C-3"', C-5"'); $170.2(\mathrm{C}=\mathrm{O}), 52.5\left(\mathrm{CH}_{2}\right), 17.8\left(2,6-\mathrm{CH}_{3}\right)$; Elem. Anal. Calcd. $\mathrm{C}_{46} \mathrm{H}_{40} \mathrm{~N}_{10} \mathrm{O}_{6}$ : C, 66.66; H, 4.86; N, 16.90; Found: C, 66.63; H, 4.81; N, 16.87; MS ES + (ToF): $m / z$ $830\left[\mathrm{M}^{+}+1\right]$.

$N, N^{\prime}-\left(6,6^{\prime}-(1,4-P h e n y l e n e) b i s(4-(4-n i t r o p h e n y l) p y r i m i-\right.$ dine-6,2-diyl))bis(2-((2,4-dimethyl phenyl)amino)acetamide) (15) Gold yellow crystals; M.p.: $135-137{ }^{\circ} \mathrm{C}$; $\mathrm{R} f$ value: 0.45 ; Yield: $89.63 \%$; IR ( $\mathrm{KBr}$ pellets, $\left.\mathrm{cm}^{-1}\right)$ : $3074(\mathrm{C}-\mathrm{H}$ str., phenyl nucleus), 1519 ( $\mathrm{C}=\mathrm{C}$ str., phenyl nucleus), 1664 ( $\mathrm{NH}-\mathrm{C}=\mathrm{O}$ str.), 1597 ( $\mathrm{N}=\mathrm{CH}$ str., of pyrimidine), 2917 (C-H str., $\mathrm{CH}_{2}$ ), 1208 (C-NH- str.), 1339 (C-N sym. str., $\left.\mathrm{Ar}-\mathrm{NO}_{2}\right) ;{ }^{1} \mathrm{H}-\mathrm{NMR}\left(\delta, \mathrm{DMSO}-d_{6}\right): 6.58-8.40\{\mathrm{~m}, 18 \mathrm{H}$, $\mathrm{Ar}=\mathrm{H}-2, \mathrm{H}-3, \mathrm{H}-5, \mathrm{H}-6,\left(\mathrm{H}-2^{\prime \prime}, \mathrm{H}-3^{\prime \prime}, \mathrm{H}-5^{\prime \prime}, \mathrm{H}-6^{\prime \prime}\right) \times 2$, (H-3"', $\left.\left.\mathrm{H}-5^{\prime \prime \prime}, \mathrm{H}-6^{\prime \prime \prime}\right) \times 2\right\}, 7.47$ (s, $2 \mathrm{H},(\mathrm{CH})_{2}$ of pyrimidine), $8.00\left(\mathrm{~s}, 2 \mathrm{H},(\mathrm{NH})_{2}\right), 3.36\left(\mathrm{~s}, 4 \mathrm{H},\left(\mathrm{CH}_{2}\right)_{2}\right), 2.51\left(\mathrm{~s}, 6 \mathrm{H},\left(\mathrm{CH}_{3}\right)_{2}\right)$; ${ }^{13} \mathrm{C}-\mathrm{NMR}\left(\delta\right.$, DMSO- $\left.d_{6}\right): 128.9$ (C-2, C-3, C-5, C-6), 136.5 (C-1, C-4); $163.0\left(\mathrm{C}-1^{\prime}, \mathrm{C}-3^{\prime}\right), 104\left(\mathrm{C}-2^{\prime}\right), 149.8\left(\mathrm{C}-5^{\prime}\right)$ pyrimidine; $141.0\left(\mathrm{C}-1^{\prime \prime}\right), 126.4\left(\mathrm{C}-2^{\prime \prime}, \mathrm{C}-6^{\prime \prime}\right), 124.5\left(\mathrm{C}-3^{\prime \prime}, \mathrm{C}-5^{\prime \prime}\right)$, 149.8 (C-4"); 143.6 (C-1"'), 126.5, 126.2 (C-2"', C-5"'), 133.9 (C-3"'), $136.9\left(\mathrm{C}-4^{\prime \prime \prime}\right), 116.1\left(\mathrm{C}-6^{\prime \prime \prime}\right) ; 188.2(\mathrm{C}=\mathrm{O}), 52.0\left(\mathrm{CH}_{2}\right)$, 18.2 $\left(o-\mathrm{CH}_{3}\right)$, $21.2\left(p-\mathrm{CH}_{3}\right)$; Elem. Anal. Calcd. $\mathrm{C}_{46} \mathrm{H}_{40} \mathrm{~N}_{10} \mathrm{O}_{6}$ : C, 66.66; H, 4.86; N, 16.90; Found: C, 66.63; H, 4.87; N, 16.95; MS ES + (ToF): $m / z 830\left[\mathrm{M}^{+}+1\right]$.

$N, N^{\prime}-\left(6,6^{\prime}-(1,4-P h e n y l e n e) b i s(4-(4-n i t r o p h e n y l) p y r i m i-\right.$ dine-6,2-diyl))bis(2-((4-bromophenyl) amino)acetamide) (16) Gold yellow crystals; M.p.: $138-140{ }^{\circ} \mathrm{C}$; $\mathrm{R} f$ value: 0.23; Yield: 87.77\%; IR (KBr pellets, $\left.\mathrm{cm}^{-1}\right): 3107(\mathrm{C}-\mathrm{H}$ str., phenyl nucleus), 1519 ( $\mathrm{C}=\mathrm{C}$ str., phenyl nucleus), 1665 ( $\mathrm{NH}-\mathrm{C}=\mathrm{O}$ str.), 1599 ( $\mathrm{N}=\mathrm{CH}$ str., of pyrimidine), 2933 (C-H str., $\mathrm{CH}_{2}$ ), 1209 (C-NH- str.), 1341 (C-N sym. str., $\left.\mathrm{Ar}-\mathrm{NO}_{2}\right), 697$ (C-Br str., Ar-Br); ${ }^{1} \mathrm{H}-\mathrm{NMR}(\delta$, DMSO$\left.d_{6}\right)$ : 6.51-8.69 $\left\{\mathrm{m}, 20 \mathrm{H}, \mathrm{Ar}=\mathrm{H}-2, \mathrm{H}-3, \mathrm{H}-5, \mathrm{H}-6,\left(\mathrm{H}-2^{\prime \prime}\right.\right.$, $\left.\left.\mathrm{H}-3^{\prime \prime}, \mathrm{H}-5^{\prime \prime}, \mathrm{H}-6^{\prime \prime}\right) \times 2,\left(\mathrm{H}-2^{\prime \prime \prime}, \mathrm{H}-3^{\prime \prime \prime}, \mathrm{H}-5^{\prime \prime \prime}, \mathrm{H}-6^{\prime \prime \prime}\right) \times 2\right\}$, $7.55\left(\mathrm{~s}, 2 \mathrm{H},(\mathrm{CH})_{2}\right.$ of pyrimidine), $8.00\left(\mathrm{~s}, 2 \mathrm{H},(\mathrm{NH})_{2}\right), 3.37$ $\left(\mathrm{s}, 4 \mathrm{H},\left(\mathrm{CH}_{2}\right)_{2}\right) ;{ }^{13} \mathrm{C}-\mathrm{NMR}\left(\delta\right.$, DMSO- $\left.d_{6}\right): 128.2(\mathrm{C}-2, \mathrm{C}-3$, C-5, C-6), 136.7 (C-1, C-4); 160.0 (C-1', C-3'), 104 (C-2'), $149.8\left(\mathrm{C}-5^{\prime}\right)$ pyrimidine; $141.5\left(\mathrm{C}-1^{\prime \prime}\right), 126.4\left(\mathrm{C}-2^{\prime \prime}, \mathrm{C}-6^{\prime \prime}\right)$, $123.5\left(\mathrm{C}-3^{\prime \prime}, \mathrm{C}-5^{\prime \prime}\right), 149.7\left(\mathrm{C}-4^{\prime \prime}\right) ; 145.5\left(\mathrm{C}-1^{\prime \prime \prime}\right), 115\left(\mathrm{C}-2^{\prime \prime \prime}\right.$, C-6"' $), 132.0$ (C-3"', C-5'"'), $118.8\left(\mathrm{C}-5^{\prime \prime \prime}\right) ; 188.2(\mathrm{C}=\mathrm{O})$, $52.5\left(\mathrm{CH}_{2}\right)$; Elem. Anal. Calcd. $\mathrm{C}_{42} \mathrm{H}_{30} \mathrm{Br}_{2} \mathrm{~N}_{10} \mathrm{O}_{6}$ : C, 54.21; H, 3.25; N, 15.05; Found: C, 54.18; H, 3.20; N, 15.00; MS $\mathrm{ES}+(\mathrm{ToF}): m / z 933\left[\mathrm{M}^{+}+1\right]$.

$N, N^{\prime}-(6,6$ - $-(1,4-$ Phenylene)bis(4-(4-nitrophenyl)pyrimidine-6,2-diyl))bis(2-((2,3-dichlorophenyl) amino)aceta- 
mide) (17) Gold yellow crystals; M.p.: $195-197{ }^{\circ} \mathrm{C}$; $\mathrm{R} f$ value: 0.16 ; Yield: $72.01 \%$; IR ( $\mathrm{KBr}$ pellets, $\left.\mathrm{cm}^{-1}\right)$ : 3108 ( $\mathrm{C}-\mathrm{H}$ str., phenyl nucleus), 1520 ( $\mathrm{C}=\mathrm{C}$ str., phenyl nucleus), 1666 ( $\mathrm{NH}-\mathrm{C}=\mathrm{O}$ str.), 1599 ( $\mathrm{N}=\mathrm{CH}$ str., of pyrimidine), 2934 (C-H str., $\mathrm{CH}_{2}$ ), 1211 (C-NH- str.), 1341 (C-N sym. str., $\left.\mathrm{Ar}-\mathrm{NO}_{2}\right), 700$ (C-Cl str., $\left.\mathrm{Ar}-\mathrm{Cl}\right)$; ${ }^{1} \mathrm{H}-\mathrm{NMR}\left(\delta, \mathrm{DMSO}-d_{6}\right): 7.93-8.39\{\mathrm{~m}, 18 \mathrm{H}, \mathrm{Ar}=\mathrm{H}-2$, $\mathrm{H}-3, \mathrm{H}-5, \mathrm{H}-6,\left(\mathrm{H}-2^{\prime \prime}, \mathrm{H}-3^{\prime \prime}, \mathrm{H}-5^{\prime \prime}, \mathrm{H}-6^{\prime \prime}\right) \times 2$, (H-4"', $\mathrm{H}-5^{\prime \prime \prime}$, $\left.\left.\mathrm{H}-6^{\prime \prime \prime}\right) \times 2\right\}, 7.91\left(\mathrm{~s}, 2 \mathrm{H},(\mathrm{CH})_{2}\right.$ of pyrimidine), $8.00(\mathrm{~s}, 2 \mathrm{H}$, $\left.(\mathrm{NH})_{2}\right), 3.38\left(\mathrm{~s}, 4 \mathrm{H},\left(\mathrm{CH}_{2}\right)_{2}\right) ;{ }^{13} \mathrm{C}-\mathrm{NMR}\left(\delta\right.$, DMSO- $\left.d_{6}\right)$ : 128.9 (C-2, C-3, C-5, C-6), 135.1 (C-1, C-4); 162.0 (C-1', C-3'), $104.1\left(\mathrm{C}-2^{\prime}\right), 149.0\left(\mathrm{C}-5^{\prime}\right)$ pyrimidine; $126.4\left(\mathrm{C}-2^{\prime \prime}\right.$, C-6"), 123.8 (C-3", C-5"), $149.6\left(\mathrm{C}-4^{\prime \prime}\right) ; 145.3$ (C-1"' $)$, 123.6 (C-2 $\left.2^{\prime \prime \prime}\right), 133.9\left(\mathrm{C}-3^{\prime \prime \prime}\right), 122.9\left(\mathrm{C}-4^{\prime \prime \prime}\right), 129.1\left(\mathrm{C}-5^{\prime \prime \prime}\right)$, $113.1\left(\mathrm{C}-6^{\prime \prime \prime}\right) ; 188.2(\mathrm{C}=\mathrm{O}), 52.6\left(\mathrm{CH}_{2}\right)$; Elem. Anal. Calcd. $\mathrm{C}_{42} \mathrm{H}_{28} \mathrm{Cl}_{4} \mathrm{~N}_{10} \mathrm{O}_{6}: \mathrm{C}, 55.40 ; \mathrm{H}, 3.10 ; \mathrm{N}, 15.38$; Found: $\mathrm{C}$, 55.38; H, 3.07; N, 15.33; MS ES + (ToF): $m / z 912\left[\mathrm{M}^{+}+1\right]$.

$N, N^{\prime}-\left(6,66^{\prime}-(1,4-P h e n y l e n e) b i s(4-(4-n i t r o p h e n y l) p y r i m i-\right.$ dine-6,2-diyl))bis(2-((2,6-dichlorophenyl) amino)acetamide) (18) Gold yellow crystals; M.p.: $129-131{ }^{\circ} \mathrm{C}$; $\mathrm{Rf}$ value: 0.33 ; Yield: $75.30 \%$; IR ( $\mathrm{KBr}$ pellets, $\mathrm{cm}^{-1}$ ): 3107 (C-H str., phenyl nucleus), 1520 ( $\mathrm{C}=\mathrm{C}$ str., phenyl nucleus), 1666 ( $\mathrm{NH}-\mathrm{C}=\mathrm{O}$ str.), 1599 ( $\mathrm{N}=\mathrm{CH}$ str., of pyrimidine), 2936 (C-H str., $\mathrm{CH}_{2}$ ), 1211 (C-NH- str.), 1342 (C-N sym. str., $\left.\mathrm{Ar}-\mathrm{NO}_{2}\right), 700$ (C-Cl str., $\left.\mathrm{Ar}-\mathrm{Cl}\right)$; ${ }^{1} \mathrm{H}-\mathrm{NMR}\left(\delta, \mathrm{DMSO}-d_{6}\right)$ : 7.04-9.09 $\{\mathrm{m}, 18 \mathrm{H}, \mathrm{Ar}=\mathrm{H}-2$, $\mathrm{H}-3, \mathrm{H}-5, \mathrm{H}-6,\left(\mathrm{H}-2^{\prime \prime}, \mathrm{H}-3^{\prime \prime}, \mathrm{H}-5^{\prime \prime}, \mathrm{H}-6^{\prime \prime}\right) \times 2$, (H-3"', H-4"', $\left.\left.\mathrm{H}-5^{\prime \prime \prime}\right) \times 2\right\}, 7.75\left(\mathrm{~s}, 2 \mathrm{H},(\mathrm{CH})_{2}\right.$ of pyrimidine), $8.01(\mathrm{~s}, 2 \mathrm{H}$, $\left.(\mathrm{NH})_{2}\right), 3.38\left(\mathrm{~s}, 4 \mathrm{H},\left(\mathrm{CH}_{2}\right)_{2}\right) ;{ }^{13} \mathrm{C}-\mathrm{NMR}\left(\delta, \mathrm{DMSO}-d_{6}\right)$ : 127.3 (C-2, C-3, C-5, C-6), 134.5 (C-1, C-4); 164.0 (C-1', $\left.\mathrm{C}-3^{\prime}\right), 102\left(\mathrm{C}-2^{\prime}\right), 148.3\left(\mathrm{C}-5^{\prime}\right)$ pyrimidine; $126.4\left(\mathrm{C}-2^{\prime \prime}\right.$, C-6" $\left.{ }^{\prime \prime}\right), 124.5\left(\mathrm{C}-3^{\prime \prime}, \mathrm{C}-5^{\prime \prime}\right), 148.6\left(\mathrm{C}-4^{\prime \prime}\right) ; 144.5\left(\mathrm{C}-1^{\prime \prime \prime}\right), 123.5$ (C-2"', C-6"'), 128.5 (C-3"', C-5"'), 121.1 (C-6"'); 165.2 $(\mathrm{C}=\mathrm{O}), 53.0\left(\mathrm{CH}_{2}\right)$; Elem. Anal. Calcd. $\mathrm{C}_{42} \mathrm{H}_{28} \mathrm{Cl}_{4} \mathrm{~N}_{10} \mathrm{O}_{6}$ : C, 55.40; H, 3.10; N, 15.38; Found: C, 76.13; H, 4.24; N, 11.12; MS ES + (ToF): $m / z 912\left[\mathrm{M}^{+}+1\right]$.

$N, N^{\prime}-(6,6$ - $-(1,4-P h e n y l e n e) b i s(4-(4-n i t r o p h e n y l) p y r i m-$ idine-6,2-diyl))bis(2-((3-methoxyphenyl) amino)acetamide) (19) Gold yellow crystals; M.p.: $198-200{ }^{\circ} \mathrm{C}$; $\mathrm{Rf}$ value: 0.21 ; Yield: $75.30 \%$; IR ( $\mathrm{KBr}$ pellets, $\left.\mathrm{cm}^{-1}\right): 3074$ (C-H str., phenyl nucleus), 1519 ( $\mathrm{C}=\mathrm{C}$ str., phenyl nucleus), 1666 ( $\mathrm{NH}-\mathrm{C}=\mathrm{O}$ str.), 1598 ( $\mathrm{N}=\mathrm{CH}$ str., of pyrimidine), 2836 (C-H str., $\mathrm{CH}_{2}$ ), 1210 (C-NH- str.), 1341 (C-N sym. str., $\left.\mathrm{Ar}-\mathrm{NO}_{2}\right), 1105$ (C-O-C str., $\left.\mathrm{Ar}-\mathrm{OCH}_{3}\right) ;{ }^{1} \mathrm{H}-$ $\operatorname{NMR}\left(\delta, \mathrm{DMSO}-d_{6}\right): 5.22-8.69\{\mathrm{~m}, 20 \mathrm{H}, \mathrm{Ar}=\mathrm{H}-2, \mathrm{H}-3$, $\mathrm{H}-5, \mathrm{H}-6,\left(\mathrm{H}-2^{\prime \prime}, \mathrm{H}-3^{\prime \prime}, \mathrm{H}-5^{\prime \prime}, \mathrm{H}-6^{\prime \prime}\right) \times 2$, (H-2'"', H-4'"', $\left.\left.\mathrm{H}-5^{\prime \prime \prime}, \mathrm{H}-6^{\prime \prime \prime}\right) \times 2\right\}, 7.41\left(\mathrm{~s}, 2 \mathrm{H},(\mathrm{CH})_{2}\right.$ of pyrimidine), 8.01 (s, $\left.2 \mathrm{H},(\mathrm{NH})_{2}\right), 3.73\left(\mathrm{~s}, 4 \mathrm{H},\left(\mathrm{CH}_{2}\right)_{2}, 3.38\left(\mathrm{~s}, 6 \mathrm{H},\left(\mathrm{OCH}_{3}\right)_{2}\right)\right.$; ${ }^{13} \mathrm{C}-\mathrm{NMR}\left(\delta, \mathrm{DMSO}-d_{6}\right): 128.3$ (C-2, C-3, C-5, C-6), 136.7 (C-1, C-4); 160.0 (C-1', C-3'), 106 (C-2'), 149.8 (C-5') pyrimidine; $141.5\left(\mathrm{C}-1^{\prime \prime}\right), 126.4\left(\mathrm{C}-2^{\prime \prime}, \mathrm{C}-6^{\prime \prime}\right), 123.5\left(\mathrm{C}-3^{\prime \prime}\right.$,
C-5 $\left.5^{\prime \prime}\right), 148.3\left(\mathrm{C}-4^{\prime \prime}\right) ; 149.7$ (C-1"' $), 106.4\left(\mathrm{C}-2^{\prime \prime \prime}, \mathrm{C}-6^{\prime \prime \prime}\right), 113.3$ $\left(\mathrm{C}-5^{\prime \prime \prime}\right) ; 188.2(\mathrm{C}=\mathrm{O}), 54.4\left(\mathrm{CH}_{2}\right), 55.1\left(\mathrm{OCH}_{3}\right)$; Elem. Anal. Calcd. $\mathrm{C}_{44} \mathrm{H}_{36} \mathrm{~N}_{10} \mathrm{O}_{8}$ : C, 63.46; H, 4.36; N, 16.82; Found: $\mathrm{C}$, 63.39; H, 4.30; N, 16.86; MS ES + (ToF): $m / z 834\left[\mathrm{M}^{+}+1\right]$.

$N, N^{\prime}-\left(6,6^{\prime}-(1,4-P h e n y l e n e) b i s(4-(4-n i t r o p h e n y l) p y r i m-\right.$ idine-6,2-diyl))bis(2-(2-methoxyphenyl) acetamide (20) Canary yellow crystals; M.p.: $179-180{ }^{\circ} \mathrm{C}$; $\mathrm{R} f$ value: 0.32; Yield: $89.23 \%$; IR (KBr pellets, $\mathrm{cm}^{-1}$ ): 3076 (C-H str., phenyl group), 1519 ( $\mathrm{C}=\mathrm{C}$ str., phenyl group), 1666 (NH$\mathrm{C}=\mathrm{O}$ str.), 1599 ( $\mathrm{N}=\mathrm{CH}$ str., of pyrimidine), $2842(\mathrm{C}-\mathrm{H}$ str., $\mathrm{CH}_{2}$ ), 1211 (C-NH- str.), 1340 (C-N sym. str., Ar$\mathrm{NO}_{2}$ ), 1107 (C-O-C str., Ar-OCH $\left.{ }_{3}\right) ;{ }^{1} \mathrm{H}-\mathrm{NMR}(\delta$, DMSO$\left.d_{6}\right)$ : 7.14-8.39 $\left\{\mathrm{m}, 20 \mathrm{H}, \mathrm{Ar}=\mathrm{H}-2, \mathrm{H}-3, \mathrm{H}-5, \mathrm{H}-6,\left(\mathrm{H}-2^{\prime \prime}\right.\right.$, $\left.\left.\mathrm{H}-3^{\prime \prime}, \mathrm{H}-5^{\prime \prime}, \mathrm{H}-6^{\prime \prime}\right) \times 2,\left(\mathrm{H}-3^{\prime \prime \prime}, \mathrm{H}-4^{\prime \prime \prime}, \mathrm{H}-5^{\prime \prime \prime}, \mathrm{H}-6^{\prime \prime \prime}\right) \times 2\right\}$, $7.47\left(\mathrm{~s}, 2 \mathrm{H},(\mathrm{CH})_{2}\right.$ of pyrimidine), $8.00\left(\mathrm{~s}, 2 \mathrm{H},(\mathrm{NH})_{2}\right)$, $3.82\left(\mathrm{~s}, 4 \mathrm{H},\left(\mathrm{CH}_{2}\right)_{2}\right), 3.75\left(\mathrm{~s}, 6 \mathrm{H},\left(\mathrm{OCH}_{3}\right)_{2}\right) ;{ }^{13} \mathrm{C}-\mathrm{NMR}(\delta$, DMSO- $\left.d_{6}\right)$ : 128.9 (C-2, C-3, C-5, C-6), 136.7 (C-1, C-4); $164.0\left(\mathrm{C}-1^{\prime}, \mathrm{C}-3^{\prime}\right), 110.0\left(\mathrm{C}-2^{\prime}\right), 149.8\left(\mathrm{C}-5^{\prime}\right)$ pyrimidine; $141.5\left(\mathrm{C}-1^{\prime \prime}\right), 126.4\left(\mathrm{C}-2^{\prime \prime}, \mathrm{C}-6^{\prime \prime}\right), 124.5\left(\mathrm{C}-3^{\prime \prime}, \mathrm{C}-5^{\prime \prime}\right), 149.7$ $\left(\mathrm{C}-4^{\prime \prime}\right)$; $126.5\left(\mathrm{C}-1^{\prime \prime \prime}\right), 158.0\left(\mathrm{C}-2^{\prime \prime \prime}\right), 55.5\left(\mathrm{OCH}_{3}\right), 113.3$ (C-3"'), 128.9 (C-4'"'), 121.3 (C-5'"'), 133.2 (C-6"' $) ; 188.2$ $(\mathrm{C}=\mathrm{O}), 39.0\left(\mathrm{CH}_{2}\right)$; Elem. Anal. Calcd. $\mathrm{C}_{44} \mathrm{H}_{36} \mathrm{~N}_{10} \mathrm{O}_{8}: \mathrm{C}$, 63.46; H, 4.36; N, 16.82; Found: C, 63.40; H, 4.31; N, 16.85; MS ES + (ToF): $m / z 834\left[\mathrm{M}^{+}+1\right]$.

\section{Biological study (antimicrobial and anticancer)}

The antimicrobial activity i.e. The minimum inhibitory concentration (MIC) of the synthesized compounds (1-20) was determined by tube dilution method [26] using cefadroxil (antibacterial) and fluconazole (antifungal) as reference drugs against Gram-positive [S. aureus, MTCC-3160 (Microbial Type Culture Collection); $B$. subtilis, MTCC-441] and Gram-negative bacteria (E. coli, MTCC-443; P. aeruginosa, MTCC-3542; S. enterica, MTCC-1165). The antifungal activity was assayed against yeast ( $C$. albicans, MTCC-227) and mould ( $A$. niger, MTCC-281). Serial dilutions of the test compounds and reference drugs were prepared in double strength nutrient broth I.P. (bacteria) or sabouraud dextrose broth I.P. (fungi) [27]. The stock solution of the test compounds and reference drugs was prepared in dimethyl sulfoxide (DMSO). Further progressive dilutions were done to obtain final concentrations of 50, 25, 12.5, 6.25, 3.125 and $1.562 \mu \mathrm{g} / \mathrm{mL}$. The samples were incubated at $37 \pm 1{ }^{\circ} \mathrm{C}$ for $24 \mathrm{~h}$ (bacteria), at $25 \pm 1{ }^{\circ} \mathrm{C}$ for 7 days (A. niger) and at $37 \pm 1{ }^{\circ} \mathrm{C}$ for $48 \mathrm{~h}$ (C. albicans) respectively and the results were recorded in terms of MIC. The MIC was the lowest concentration of the tested compound that yields no visible growth of microorganisms in the tube. To ensure that the solvent had no effect on the bacterial growth, a control was performed with the test medium supplemented with DMSO at the same dilutions as 
used in the experiments and DMSO had no effect on the microorganisms in the concentrations studied. The anticancer screening $\left(\mathrm{IC}_{50}=\mu \mathrm{mol} / \mathrm{mL}\right)$ of synthesized compounds was determined against human colorectal carcinoma [HCT-116 (ATCC (American Type Culture Collection) CCL-247)] cancer cell line using sulforhodamine-B (SRB) assay. In this study, the culture material was fixed with trichloroacetic acid and then stained for 30 min with $0.4 \%(\mathrm{w} / \mathrm{v})$ sulforhodamine B mixed with $1 \%$ acetic acid. Unbound dye was discarded by five washes of $1 \%$ acetic acid solution and protein-bound dye was extracted with $10 \mathrm{mM}$ Tris base [tris(hydroxymethyl) aminomethane] for confirmation of optical density in a computer-interfaced, 96-well microtiter plate reader [28].

\section{Conclusion}

In summary, a series of new bis-pyrimidine acetamide molecules was synthesized in good yields and its chemical structures were confirmed by ${ }^{1} \mathrm{H} /{ }^{13} \mathrm{C}$-NMR, Mass, FT-IR studies and elemental analyses. All the synthesized compounds were tested for their in vitro antimicrobial and anticancer potentials. Among the synthesized compounds, compounds, 3, 13, 16, 17 and 18 exhibited good antimicrobial potential against different microorganism (bacterial species: S. aureus, B. subtilis, E. coli, P. aeruginosa, S. enterica and fungal species: A. niger and C. albicans) than the standard drugs cefadroxil and fluconazole. Similarly, compounds, $\mathbf{1 2}, \mathbf{1 6}$ and $\mathbf{1 8}$ were found to be more effective against HCT 116 cancer cell line than the standard drug, 5-fluorouracil.

\section{Authors' contributions \\ $B N$ and SK have designed, synthesized and carried out the antimicrobial activ- ity of the bis-pyrimidine acetamide derivatives. KR, SAAS, SML and VM have carried out the spectral analysis, interpretation and anticancer evaluation of synthesized compounds. All authors read and approved the final manuscript.}

\section{Author details}

${ }^{1}$ Faculty of Pharmaceutical Sciences, Maharshi Dayanand University, Rohtak 124001, India. ${ }^{2}$ Department of Pharmaceutical Sciences, Maharshi Dayanand University, Rohtak 124001, India. ${ }^{3}$ Faculty of Pharmacy, Universiti. Teknologi MARA (UiTM), 42300 Bandar Puncak Alam, Selangor Darul Ehsan, Malaysia. ${ }^{4}$ Collaborative Drug Discovery Research (CDDR) Group, Pharmaceutical Life Sciences Community of Research, Universiti Teknologi MARA (UiTM), 40450 Shah Alam, Selangor Darul Ehsan, Malaysia. ${ }^{5}$ Department of Pharmacology and Toxicology, College of Pharmacy, Qassim University, Buraidah 51452, Kingdom of Saudi Arabia. ${ }^{6}$ Atta-ur-Rahman Institute for Natural Products Discovery (AuRIns), Universiti Teknologi MARA, Puncak Alam Campus, 42300 Bandar Puncak Alam, Selangor D. E, Malaysia.

\section{Acknowledgements}

The authors are thankful to Head, Department of Pharmaceutical Sciences, M. D. University, Rohtak for providing necessary facilities to carry out this research work.

\section{Competing interests}

The authors declare that they have no competing interests.

Ethics approval and consent to participate Not applicable.

\section{Publisher's Note}

Springer Nature remains neutral with regard to jurisdictional claims in published maps and institutional affiliations.

Received: 22 June 2017 Accepted: 1 August 2017

Published online: 08 August 2017

\section{References}

1. Keche AP, Hatnapure GD, Tale RH, Rodge AH, Birajdar SS, Kamble VM (2012) Novel pyrimidine derivatives with aryl urea, thiourea and sulfonamide moieties: synthesis, anti-inflammatory and antimicrobial evaluation. Bioorg Med Chem Lett 22:3445-3448

2. Zijian L, Yu W, Lin L, Dazhuang Z, Lihui W, Yanfang Z, Ping G (2014) Design synthesis and biological evaluation of novel thieno $[3,2-d]$ pyrimidine derivatives containing diaryl urea moiety as potent antitumor agents. Eur J Med Chem 85:215-227

3. Anupama B, Dinda SC, Prasad YR, Rao AV (2012) Synthesis and antimicrobial activity of some new 2,4,6-trisubstituted pyrimidines. Int J Res Pharm Chem 2(2):231-236

4. Yerragunta V, Patil P, Anusha V, Swamy TK, Suman D, Samhitha T (2013) Pyrimidine and its biological activity: a review. Pharma Tutor 1(2):39-44

5. Holla BS, Mahalinga M, Karthikeyan MS, Akberalib PM, Shettyc NS (2006) Synthesis of some novel pyrazolo $[3,4-d]$ pyrimidine derivatives as potential antimicrobial agents. Bioorg Med Chem 14:2040-2047

6. El-Gaby MSA, Gaber AM, Atalla AA, Al-Wahab KAA (2002) Novel synthesis and antifungal activity of pyrrole and pyrrolo [2,3-d] pyrimidine derivatives containing sulfonamide moieties. II Farmaco 57:613-617

7. Pandey S, Suryawanshi SN, Gupta S, Srivastava VML (2004) Synthesis and antileishmanial profile of some novel terpenyl pyrimidines. Eur J Med Chem 39:969-973

8. Sharma V, Chitranshi N, Agarwal AK (2014) Significance and biological importance of pyrimidine in the microbial world. Int J Med Chem 2014:1-31

9. Chen PJ, Yang A, GU YF, Zhang XS, Shao KP, Xue DQ, He P, Jiang TF, Zhang QR, Liu HM (2014) Synthesis, in vitro antimicrobial and cytotoxic activities of novel pyrimidine benzimidazol combinations. Bioorg Med Chem Lett 24:2741-2743

10. Ashour HM, Shaaban OG, Rizk OH, El-Ashmawy IM (2013) Synthesis and biological evaluation of thieno $\left[2^{\prime}, 3^{\prime}: 4,5\right]$ pyrimido $[1,2-b][1,2,4]$ triazines and thieno $[2,3-d][1,2,4]$ triazolo $[1,5-a]$ pyrimidines as anti-inflammatory and analgesic agents. Eur J Med Chem 62:341-351

11. Song XJ, Shao Y, Gao X (2011) Dong Microwave-assisted synthesis of some novel fluorinated pyrazolo $[3,4-d]$ pyrimidine derivatives containing 1,3,4-thiadiazole as potential antitumor agents. Chin Chem Lett 22:1036-1038

12. Meneghesso S, Vanderlinden E, Stevaert A, McGuigan C, Balzarini J, Naesens $L$ (2012) Synthesis and biological evaluation of pyrimidine nucleoside monophosphate prodrugs targeted against influenza virus. Antivir Res 94:35-43

13. Faldu VJ, Gothalia VK, Shah VH (2015) Characterization and antitubercular activity of synthesized pyrimidine derivatives via chalcones. Indian J Chem 54B:391-398

14. Kumar D, Khan SI, Tekwani BL, Diwan PP, Rawat S (2015) 4-Aminoquinoline-pyrimidine hybrids: synthesis, antimalarial activity, heme binding and docking studies. Eur J Med Chem 89:490-502

15. Kotaiah Y, Nagaraju K, Harikrishna N, Rao CV, Yamini L, Vijjulatha M (2014) Synthesis, docking and evaluation of antioxidant and antimicrobial activities of novel 1,2,4-triazolo [3,4-b][1, 3,4] thiadiazol-6-yl) selenopheno [2,3-d] pyrimidines. Eur J Med Chem 75:195-202

16. Saini M, Kumar P, Kumar M, Kalavathy R, Mani V, Mishra RK, Majeed ABA, Narasimhan B (2014) Synthesis, in vitro antimicrobial, anticancer evaluation and QSAR studies of $N^{\prime}$-(substituted)-4-(butan-2-lideneamino) benzohydrazides. Arab J Chem 7(4):448-460

17. Deep A, Kumar P, Narasimhan B, Mishra RK, Mani V, Ramasamy K, Meng LS (2016) 2-Azetidinone derivatives: synthesis, antimicrobial, anticancer evaluation and QSAR studies. Acta Pol Pharm Drug Res 73:65-78 
18. Kumar S, Kumar P, Marwaha RK, Narasimhan B (2017) Synthesis, antimicrobial evaluation and QSAR studies of propionic acid derivatives. Arab Chem 10:S881-S893

19. Ghoneim AA, El-Farargy AF (2016) Synthesis of some novel fused heterocyclic compounds derivatives from bis-chalcones. Chem Curr Res $5(1): 1-5$

20. Kharb R, Tyagi M, Sharma AK (2014) Current status and future scenario of pyrimidine derivatives having antimicrobial potential. Der Pharma Chem 6(4):298-320

21. Yaziji V, Rodríguez D, Coelho A, García-Mera X, El Maatougui A, Brea J, Loza MI, Cadavid MI, Gutiérrez-de-Terán H, Sotelo E (2013) Selective and potent adenosine $\mathrm{A} 3$ receptor antagonists by methoxyaryl substitution on the N-(2,6-diarylpyrimidin-4-yl) acetamide scaffold. Eur J Med Chem 59:235-242

22. Kanagarajan V, Thanusu J, Gopalakrishnan M (2010) Synthesis and in vitro microbiological evaluation of an array of biolabile 2-morpholino- $\mathrm{N}-(4,6-$ diaryl pyrimidin-2-yl) acetamides. Eur J Med Chem 45:1583-1589
23. Asiri AM, Khan SA (2011) Synthesis and anti-bacterial activities of a bis-chalcone, derived from thiophene and its bis-cyclized products. Molecules 16:523-531

24. Parveen H, Hayat F, Mukhtar S, Salahuddin A, Khan A, Islam F, Azam A (2011) Synthesis, characterization and biological evaluation of novel 2,4,6-trisubstituted bis-pyrimidine derivatives. Eur J Med Chem 46:4669-4675

25. Naik TA, Chikhalia KH (2007) Studies on synthesis of pyrimidine derivatives and their pharmacological evaluation. E-J Chem 4(1):60-66

26. Cappuccino JC, Sherman N (1999) Microbiology—a laboratory manual. Addison Wesley, California, p 263

27. Pharmacopoeia of India, vol I (2007) Controller of publication, Ministry of Health Department, Govt. of India, New Delhi, pp. 37

28. Skehan P, Storeng Scudiero RD, Monks A, McMahon J, Vistica D, Warren JT, Bokesch H, Kenney S, Boyd MR (1990) New colorimetric cytotoxicity assay for anticancer-drug screening. J Natl Cancer Inst 82:1107-1112

\section{Submit your manuscript to a SpringerOpen ${ }^{\odot}$ journal and benefit from:}

- Convenient online submission

- Rigorous peer review

- Open access: articles freely available online

- High visibility within the field

- Retaining the copyright to your article

Submit your next manuscript at $\gg$ springeropen.com 